\title{
A Burgess-like subconvex bound for twisted $L$-functions
}

\author{
V. Blomer, G. Harcos, P. Michel, and Appendix 2 by Z. Mao \\ (Communicated by Peter Sarnak)
}

\begin{abstract}
Let $g$ be a cuspidal newform (holomorphic or Maass) of arbitrary level and nebentypus, $\chi$ a primitive character of conductor $q$, and $s$ a point on the critical line $\Re s=\frac{1}{2}$. It is proved that$$
L(g \otimes \chi, s) \ll_{\varepsilon, g, s} q^{1 / 2-(1 / 8)(1-2 \theta)+\varepsilon},
$$

where $\varepsilon>0$ is arbitrary and $\theta=\frac{7}{64}$ is the current known approximation towards the Ramanujan-Petersson conjecture (which would allow $\theta=0$ ); moreover, the dependence on $s$ and all the parameters of $g$ is polynomial. This result is an analog of Burgess' classical subconvex bound for Dirichlet $L$-functions. In Appendix 2 the above result is combined with a theorem of Waldspurger and the adelic calculations of Baruch-Mao to yield an improved uniform upper bound for the Fourier coefficients of holomorphic half-integral weight cusp forms.
\end{abstract}

2000 Mathematics Subject Classification: 11F66; 11F67, $11 \mathrm{M} 41$.

\section{Introduction}

Let $g$ be a general cusp form, that is,

- a holomorphic form of integral weight $k_{g} \geq 1$, level $D$, and nebentypus $\chi_{g}$,

- or a Maass form of weight 0 or 1 (without loss of generality), level $D$, and nebentypus $\chi_{g}$ having spectral parameter $t_{g}=\left(\lambda-\frac{1}{4}\right)^{1 / 2}$, where $\lambda$ is the Laplacian eigenvalue.

We suppose that $g$ is "new" in the sense of Atkin-Lehner theory; in particular, $g$ is an eigenform of the Hecke operators $T_{n}, n \geq 1$, and we will denote by $\lambda_{g}(n)$ its $n$-th Hecke eigenvalue. We shall be concerned with the twist $g \otimes \chi$, where $\chi$ is a primitive

The third author was supported by the Institut Universitaire de France and by the ACI "Arithmétique des fonctions $L$ ".

The fourth author was partially supported by NSF grant DMS-0355285. 
character to a large modulus $q$. This is again a newform (of level dividing $D q^{2}$ ), and its $L$-function $L(g \otimes \chi, s)$ equals up to finitely many Euler factors $\sum_{n} \lambda_{g}(n) \chi(n) n^{-s}$; to be precise, we have

$$
\begin{aligned}
& L(g \otimes \chi, s)=\sum_{n \geq 1} \frac{\lambda_{g \otimes \chi}(n)}{n^{s}} \\
& =\prod_{p \nmid(q, D)}\left(1-\frac{\lambda_{g}(p) \chi(p)}{p^{s}}+\frac{\chi^{2} \cdot \chi_{g}(p)}{p^{2 s}}\right)^{-1} \prod_{p \mid(q, D)}\left(1-\frac{\lambda_{g \otimes \chi}(p)}{p^{s}}+\frac{\chi_{g \otimes \chi}(p)}{p^{2 s}}\right)^{-1} .
\end{aligned}
$$

In this paper we are interested in upper bounds for $L(g \otimes \chi, s)$ when $s$ is on the critical line $\Re s=\frac{1}{2}$.

In some respects, the weight $k_{g}$ of a holomorphic form and the spectral parameter $t_{g}$ of a Maass form behave similarly, the reason being that if $g$ is holomorphic of weight $k$, then $y^{k / 2} g(z)$ is Maass having spectral parameter $t=i \frac{k-1}{2}$. For a uniform notation let us therefore define the infinity type $\tilde{\mu}_{g}$ of $g$ as

$$
\tilde{\mu}_{g}:= \begin{cases}\frac{k_{g}-1}{2} & \text { if } g \text { is holomorphic of weight } k_{g} \\ t_{g} & \text { if } g \text { is a Maass form of weight } 0 \text { or } 1 \text { and } \\ & \text { Laplacian eigenvalue } \frac{1}{4}+t_{g}^{2}\end{cases}
$$

and let us write $\mu_{g}:=1+\left|\tilde{\mu}_{g}\right|$. Then the general convexity bound gives

$$
L(g \otimes \chi, s) \ll_{\varepsilon}\left(|s| \mu_{g} q D\right)^{\varepsilon}\left(|s| \mu_{g}\right)^{1 / 2} D^{1 / 4} q^{1 / 2}
$$

for $\Re s=\frac{1}{2}$ and for any $\varepsilon>0$ which, however, is often not sufficient for applications. In particular, it is of interest to break convexity in the $q$-aspect while keeping a polynomial control in the remaining parameters $|s|, \mu_{g}, D$.

The first breakthrough was obtained by Duke-Friedlander-Iwaniec [DFI93]. If $g$ is holomorphic of level $D=1$ they proved the subconvex exponent

$$
\text { (1.2) } \frac{1}{2}-\frac{1}{22}
$$

using the $\delta$-symbol method. In the case of a general holomorphic cusp form of weight at least 2, Bykovskiı [By96] derived, by a different method, the stronger subconvex exponent

$$
\frac{1}{2}-\frac{1}{8}
$$

as long as $(D, q)=1$. While it is unclear whether and to what extent Bykovskiı̌'s method carries over to the general case (1.1), the second and third author in- 
dependently used the strategy from [DFI93] to break convexity also in the Maass case $[\mathrm{H} 03 \mathrm{a}, \mathrm{H} 03 \mathrm{~b}, \mathrm{M} 04 \mathrm{a}]$. As a notable feature of [H03a], a very flexible variant of the $\delta$-symbol method due to Jutila $[\mathrm{J92}, \mathrm{J} 96, \mathrm{~J} 99]$ was introduced into the argument.

Sarnak [S01] recently developed a new method using relatively deep spectral analysis and in particular estimates for triple products of automorphic forms. Although not stated explicitly, his method yields

$$
\frac{1}{2}-\frac{1-2 \theta}{14+4 \theta}
$$

when $g$ is holomorphic; see also [Co03, CoPSS] for an explicit version in the more general context of holomorphic modular forms over totally real fields. This is stronger than (1.2), but weaker than (1.3). Here and henceforth, $\theta \geq 0$ denotes any admissible constant, by which we mean that the following approximation to the Generalized Ramanujan-Petersson conjecture is satisfied:

Hypothesis $\boldsymbol{H}_{\boldsymbol{\theta}}$. For any cuspidal automorphic representation $\pi$ on $\mathrm{GL}_{2}(\mathbb{Q}) \backslash \mathrm{GL}_{2}\left(\mathbb{A}_{\mathbb{Q}}\right)$ with local Hecke parameters $\alpha_{\pi}^{(1)}(p), \alpha_{\pi}^{(2)}(p)$ for $p<\infty$ and $\mu_{\pi}^{(1)}(\infty), \mu_{\pi}^{(2)}(\infty)$, one has the bounds

$$
\begin{aligned}
& \left|\alpha_{\pi}^{(j)}(p)\right| \leq p^{\theta}, \quad j=1,2 \\
& \left(\text { resp. }\left|\Re \mu_{\pi}^{(j)}(\infty)\right| \leq \theta, j=1,2\right)
\end{aligned}
$$

provided $\pi_{p}$ (resp. $\left.\pi_{\infty}\right)$ is unramified.

Currently, the best admissible constant is $\theta=\frac{7}{64}$ as follows from the work of Shahidi, Kim-Shahidi, Kim and Kim-Sarnak [KS02, K03, KS03].

Eventually, Sarnak's method and the bound (1.4) can be generalized to arbitrary $g$, but this requires very delicate arguments from the theory of automorphic representations. However, these difficulties can be avoided, and in this paper we combine various ideas from [B104b, HM04b] to obtain the stronger exponent

$$
\frac{1}{2}-\frac{1-2 \theta}{8}
$$

valid in the general case (1.1). Precisely, we have

Theorem 1. Let $g$ be a cuspidal automorphic newform (i.e., either a holomorphic form or a Maass cusp form) as in (1.1), and let $\chi$ be a primitive character of conductor $q$. For any $\varepsilon>0$ and for $\Re s=\frac{1}{2}$ one has

$$
L(g \otimes \chi, s) \ll_{\varepsilon}\left(|s| \mu_{g} D q\right)^{\varepsilon}|s|^{A} \mu_{g}^{B} D^{C} q^{1 / 2-(1 / 8)(1-2 \theta)},
$$


where

$$
A:=\frac{31+4 \theta}{16}, \quad B:=\frac{75+12 \theta}{16}, \quad C:=\frac{9}{16},
$$

and $0 \leq \theta<\frac{1}{2}$ is any real number such that Hypothesis $H_{\theta}$ is satisfied. Since $\theta=\frac{7}{64}$ is currently admissible, the current subconvex exponent equals

$$
\frac{1}{2}-\frac{25}{256}=\frac{103}{256}=0.40234375
$$

Remark 1.1. Unlike all previous bounds, our estimate is explicit in all the parameters of $g$ which turns out to be useful for applications. The numerical values of $A, B, C$ can be improved with more careful estimates.

Remark 1.2. The bound (1.6) should be compared with Burgess' bound [B63] for Dirichlet $L$-functions. Indeed, the square of $L(\chi, s)$ can be interpreted as the twisted $L$-function $L(E \otimes \chi, s)$, where $E$ denotes the (derivative of the) standard weight zero Eisenstein series of level 1 and Laplacian eigenvalue $\frac{1}{4}$. In this context, Burgess' bound (in its hybrid version by Heath-Brown [HB78]) is written as

$$
L(E \otimes \chi, s) \ll_{\varepsilon}(|s| q)^{\varepsilon}|s|^{1 / 2} q^{1 / 2-1 / 8} .
$$

Thus the bound of Theorem 1 is the cuspidal analog of Burgess' result under the Ramanujan-Petersson conjecture (i.e., $\theta=0$ ).

Remark 1.3. Under more restricted assumptions, the sharpest subconvex exponent for this problem is due to Conrey-Iwaniec [CI00], namely

$$
L\left(g \otimes \chi, \frac{1}{2}\right) \ll_{\varepsilon, \mu_{g}} q^{1 / 3+\varepsilon}
$$

for a quadratic character $\chi$ and for $g$ either a cusp form of level 1 or $g(z)=$ $E\left(z, \frac{1}{2}+i t\right)$ the Eisenstein series of full level (here $\left.t \in \mathbb{R}\right)$; in the latter case, one has

$$
L\left(g \otimes \chi, \frac{1}{2}\right)=\left|L\left(\chi, \frac{1}{2}+i t\right)\right|^{2},
$$

so this bound is the exact analog of Weyl's $\frac{1}{6}$ bound for the Riemann zeta function. Note, however, that the argument leading to this bound uses crucially the positivity of the central value $L\left(g \otimes \chi, \frac{1}{2}\right)$ and is therefore limited to the case of $\chi$ a quadratic character, $g$ a self-dual modular form and to the special value $s=\frac{1}{2}$.

Remark 1.4. It is a nice feature that our method permits a uniform treatment of all cusp forms on $\mathrm{GL}_{2}(\mathbb{Q}) \backslash \mathrm{GL}_{2}\left(\mathbb{A}_{\mathbb{Q}}\right)$. Depending on the applications, Theorem 1 can be 
optimized with respect to various auxiliary parameters, and it can be used as an ingredient for several other subconvexity problems, some of which will be considered elsewhere. Here we want to focus on Rankin-Selberg $L$-functions. Let $f$ and $g$ be two cuspidal newforms. Then for $s$ on the critical line one has the convexity bound

$$
L(f \times g, s) \ll_{\varepsilon, \mu_{f}, g, s} q^{1 / 2+\varepsilon},
$$

where $q$ denotes the level of $f$. The problem of improving this estimate was solved in [KMV02, M04a, HM04a]. The hardest case is when the conductor of the nebentypus of $f$ is large (if the nebentypus is primitive for instance). In this configuration, the subconvexity problem for twisted $L$-functions plays a key role. In [HM04a], we use the results of the present paper to obtain the following corollary:

Corollary. There exist positive absolute constants $A, \delta>0$ with the following property. For any two newforms $f$ and $g$ (holomorphic or Maass) of respective levels $q, D$ and respective nebentypus $\chi_{f}$, $\chi_{g}$ such that $\chi_{f} \chi_{g}$ is non-trivial, one has

$$
L(f \times g, s) \ll\left(|s| \mu_{f} \mu_{g} D\right)^{A} q^{1 / 2-\delta}
$$

for $\Re s=\frac{1}{2}$. Assuming Hypothesis $H_{\theta}$ we can take

$$
\delta=\frac{(1-2 \theta)}{8} \frac{(1-2 \theta)}{202}-\varepsilon,
$$

so that at the current state of knowledge $\delta=\frac{1}{2648}$ is admissible.

Combining some of the methods of [HM04a, B104b] and of the present paper it is possible to reduce considerably the constant 202 above. We will return to this on another occasion.

Remark 1.5. Theorem 1 can be combined with the powerful results of Shimura and Waldspurger to improve on the known upper bounds for the Fourier coefficients of half-integral weight holomorphic or Maass cusp forms. The recent careful adelic calculations of Baruch-Mao enable one to derive these estimates with proper uniformity in all the parameters of the underlying cusp form. The details in the holomorphic case have been kindly worked out for us by Zhengyu Mao and have been included in this paper as Appendix 2 (see Theorem 6).

Acknowledgements. The second author wishes to thank Université Montpellier II for its hospitality during the week June 15-21, 2004. The third author would like to thank the American Institute of Mathematics (Palo-Alto) and the organizers of the workshop "Emerging applications of measure rigidity" - during which this work was initiated - for their kind invitation and the excellent working conditions. 


\section{Some general results}

In this section, we indicate our normalization for the Fourier coefficients of modular forms, recall some of their properties and state various results from the spectral theory of automorphic forms; for more background and references, we refer to [DFI02] and to [HM04a].

2.1. Fourier coefficients. We will follow the notation of [DFI02] to large extent. A Maass cusp form $g$ of weight $k \geq 0$ and Laplacian eigenvalue $\left(\frac{1}{2}+i t\right)\left(\frac{1}{2}-i t\right)$ admits an expansion

$$
g(z)=\sum_{\substack{n \in \mathbb{Z} \\ n \neq 0}} \rho_{g}(n) W_{(n /|n|)(k / 2), i t}(4 \pi|n| y) e(n x)
$$

in terms of the Whittaker function $W_{\alpha, i t}(y)$. Note that $W_{\alpha, i t}(y) \sim y^{\alpha} e^{-y / 2}$ for $y \rightarrow+\infty$. For an Eisenstein series $E_{\mathfrak{a}}\left(z, \frac{1}{2}+i t\right)$ attached to some cusp a of $\Gamma_{0}(D)$, we have a Fourier expansion of the type

$$
\begin{aligned}
E_{\mathfrak{a}}\left(z, \frac{1}{2}+i t\right)= & \delta_{\mathfrak{a}=\infty} y^{1 / 2+i t}+\varphi_{\mathfrak{a}}\left(\frac{1}{2}+i t\right) y^{1 / 2-i t} \\
& +\sum_{\substack{n \in \mathbb{Z} \\
n \neq 0}} \rho_{\mathfrak{a}}(n, t) W_{(n /|n|)(k / 2), i t}(4 \pi|n| y) e(n x) .
\end{aligned}
$$

Finally, when $g(z)$ is a holomorphic cusp form of weight $k$, we write

$$
g(z)=\sum_{n \geq 1} \rho_{g}(n)(4 \pi n)^{k / 2} e(n z),
$$

keeping in mind that $y^{k / 2} g(z)$ is a Maass form of weight $k$.

We will need the following general Voronoi-type summation formula ([HM04a, Proposition 2.1]).

Proposition 2.1. Let $g$ be a cusp form (holomorphic or Maass) of weight $k$, level $D$ and nebentypus $\chi_{g}$. Let $c \equiv 0(\bmod D)$, and let $a$ be an integer coprime to $c$. If $F \in C^{\infty}((0, \infty))$ is a Schwartz class function vanishing in a neighborhood of zero, then

$$
\sum_{n \geq 1} \sqrt{n} \rho_{g}(n) e\left(n \frac{a}{c}\right) F(n)=\frac{\chi_{g}(\bar{a})}{c} \sum_{ \pm} \sum_{n \geq 1} \sqrt{n} \rho_{g}^{ \pm}(n) e\left(\mp n \frac{\bar{a}}{c}\right) \mathscr{F} \pm\left(\frac{n}{c^{2}}\right) .
$$

In this formula,

$$
\rho_{g}^{+}(n):=\rho_{g}(n), \quad \rho_{g}^{-}(n):=\frac{\Gamma\left(\frac{1}{2}+i t-\frac{k}{2}\right)}{\Gamma\left(\frac{1}{2}+i t+\frac{k}{2}\right)} \rho_{g}(-n),
$$

and 


$$
\mathscr{F}^{ \pm}(y):=\int_{0}^{\infty} F(x) J_{g}^{ \pm}(4 \pi \sqrt{x y}) d x
$$

where $t=t_{g}$ is the spectral parameter of $g$ in the Maass case, and

$$
J_{g}^{+}(x):=2 \pi i^{l} J_{l-1}(x), \quad J_{g}^{-}(x):=0,
$$

if $g$ is induced from a holomorphic form of weight $l$;

$$
J_{g}^{+}(x):=\frac{-\pi}{\operatorname{ch}(\pi t)}\left\{Y_{2 i t}(x)+Y_{-2 i t}(x)\right\}, \quad J_{g}^{-}(x):=4 \operatorname{ch}(\pi t) K_{2 i t}(x),
$$

if $k$ is even, and $g$ is not induced from a holomorphic form;

$$
J_{g}^{+}(x):=\frac{\pi}{\operatorname{sh}(\pi t)}\left\{Y_{2 i t}(x)-Y_{-2 i t}(x)\right\}, \quad J_{g}^{-}(x):=-4 i \operatorname{sh}(\pi t) K_{2 i t}(x),
$$

if $k$ is odd, and $g$ is not induced from a holomorphic form.

2.2. Hecke operators. We recall that there is an action on the $L^{2}$-space of modular forms of level $D$ and some given nebentypus by the commutative algebra $\mathbb{T}$ generated by the Hecke operators $\left\{T_{n}\right\}_{n \geq 1}$. We denote by $\mathbb{T}^{(D)}$ the subalgebra generated by $\left\{T_{n}\right\}_{(n, D)=1}$ and call a holomorphic or Maass cusp form a Hecke-Maass cusp form if it is an eigenform for $\mathbb{T}^{(D)}$. For a Hecke-Maass cusp form $g$ we denote by $\chi_{g}$ its nebentypus and by $\lambda_{g}(n)$ its $n$-th Hecke eigenvalue. If Hypothesis $H_{\theta}$ is valid, one has

$$
\left|\lambda_{g}(n)\right| \leq \tau(n) n^{\theta}
$$

where $\tau(n)$ denote the divisor function. Moreover, for $(n, D)=1$ the following relations hold:

$$
\begin{aligned}
& \sqrt{n} \rho_{g}( \pm n)=\rho_{g}( \pm 1) \lambda_{g}(n) \\
& \sqrt{m} \rho_{g}(m) \lambda_{g}(n)=\sum_{d \mid(m, n)} \chi_{g}(d) \rho_{g}\left(\frac{m}{d} \frac{n}{d}\right) \sqrt{\frac{m n}{d^{2}}} \\
& \sqrt{m n} \rho_{g}(m n)=\sum_{d \mid(m, n)} \chi_{g}(d) \mu(d) \rho_{g}\left(\frac{m}{d}\right) \sqrt{\frac{m}{d}} \lambda_{g}\left(\frac{n}{d}\right)
\end{aligned}
$$

If $g$ is a Hecke form and belongs to the new subspace (in the sense of Atkin-Lehner theory), then $g$ an eigenform of all Hecke operators and the above relations hold with no restriction on $n$. In this case, we say that $g$ is a newform.

2.3. Kuznetsov's formula and the large sieve. We make the following convention: if $f$ and $g$ are two Maass cusp forms of the same weight, same level $D$, and same nebentypus, then we normalize their Petersson inner product $\langle f, g\rangle$ as 


$$
\langle f, g\rangle=\int_{\Gamma_{0}(D) \backslash \mathbb{H}} \bar{f}(z) g(z) \frac{d x d y}{y^{2}}
$$

if $f$ and $g$ are holomorphic of weight $k,\langle f, g\rangle$ is given as above with an extra $y^{k}$ factor. In particular, we say that $g$ is $L^{2}$-normalized if $\langle g, g\rangle=1$.

For a character $\chi$ to modulus $D$, we denote by $\mathscr{B}(D ; \chi)=\left\{f_{j}\right\}_{j \geq 1}\left(\right.$ resp. $\left.\mathscr{B}_{k}^{h}(D ; \chi)\right)$ an orthonormal basis of the $L^{2}$-space of weight 0 Maass cusp forms (resp. of the space of holomorphic cusp forms of weight $k$ ) of level $D$ and nebentypus $\chi$. If $\chi$ is the trivial character, we simply omit it from the notation. We can always choose a basis formed of Hecke-Maass cusp forms, and we will pick a special basis later in section 3.1. Let us now recall Kuznetsov's trace formula (in the trivial nebentypus case, see [I87, Theorems 9.4, 9.5, 9.7]).

Theorem 2. Let $m, n, D$ be positive integers and $\varphi \in C_{c}^{\infty}((0, \infty))$. One has

$$
\begin{aligned}
& \frac{1}{4 \sqrt{m n}} \sum_{c \equiv 0(D)} \frac{S(m, n ; c)}{c} \varphi\left(\frac{4 \pi \sqrt{m n}}{c}\right) \\
& =\sum_{k \equiv 0(2)} \Gamma(k) \tilde{\varphi}(k-1) \sum_{f \in \mathscr{B}_{k}^{h}(D)} \overline{\rho_{f}}(m) \rho_{f}(n)+\sum_{j \geq 1} \frac{\hat{\varphi}\left(t_{j}\right)}{\operatorname{ch}\left(\pi t_{j}\right)} \overline{\rho_{j}}(m) \rho_{j}(n) \\
& \quad+\frac{1}{4 \pi} \sum_{\mathfrak{a}} \int_{-\infty}^{+\infty} \frac{\hat{\varphi}(t)}{\operatorname{ch}(\pi t)} \overline{\rho_{\mathfrak{a}}}(m, t) \rho_{\mathfrak{a}}(n, t) d t,
\end{aligned}
$$

and

$$
\begin{aligned}
& \frac{1}{4 \sqrt{m n}} \sum_{c \equiv 0(D)} \frac{S(m,-n ; c)}{c} \varphi\left(\frac{4 \pi \sqrt{m n}}{c}\right) \\
& =\sum_{j \geq 1} \frac{\check{\varphi}\left(t_{j}\right)}{\operatorname{ch}\left(\pi t_{j}\right)} \bar{\rho}_{j}(m) \rho_{j}(-n)+\frac{1}{4 \pi} \sum_{\mathfrak{a}} \int_{-\infty}^{+\infty} \frac{\check{\varphi}(t)}{\operatorname{ch}(\pi t)} \overline{\rho_{\mathfrak{a}}}(m, t) \rho_{\mathfrak{a}}(-n, t) d t,
\end{aligned}
$$

where the Bessel transforms are defined by

$$
\begin{aligned}
& \tilde{\varphi}(k-1):=\int_{0}^{\infty} \varphi(x) i^{k} J_{k-1}(x) \frac{d x}{x} \\
& \hat{\varphi}(t):=\int_{0}^{\infty} \varphi(x) \frac{-\pi}{2 \operatorname{ch}(\pi t)}\left\{Y_{2 i t}(x)+Y_{-2 i t}(x)\right\} \frac{d x}{x} \\
& \check{\varphi}(t):=\int_{0}^{\infty} \varphi(x) 2 \operatorname{ch}(\pi t) K_{2 i t}(x) \frac{d x}{x} .
\end{aligned}
$$


Remark 2.1. The kernels in (2.9) and (2.10) can be expressed alternatively as

$$
\begin{aligned}
& \frac{-\pi}{2 \operatorname{ch}(\pi t)}\left\{Y_{2 i t}(x)+Y_{-2 i t}(x)\right\}=\frac{\pi i}{2 \operatorname{sh}(\pi t)}\left\{J_{2 i t}(x)-J_{-2 i t}(x)\right\} ; \\
& 2 \operatorname{ch}(\pi t) K_{2 i t}(x)=\frac{\pi i}{2 \operatorname{sh}(\pi t)}\left\{I_{2 i t}(x)-I_{-2 i t}(x)\right\} .
\end{aligned}
$$

The next lemma is a variant of Lemma 7.1 of [DI82] and provides bounds for the various Bessel transforms of the test functions $\varphi$ above.

Lemma 2.1. Let $\varphi(x)$ be a smooth function, compactly supported in $(X, 2 X)$, satisfying

$$
\varphi^{(i)}(x) \ll_{i}(Z / X)^{i}
$$

for some $Z \geq 1$ and for any integer $i \geq 0$, the implied constant depending only on $i$. Then, for $t \geq 0$ and for any real $k>1$, one has

$$
\hat{\varphi}(i t), \check{\varphi}(i t) \ll \frac{1+(X / Z)^{-2 t}}{1+X / Z} \quad \text { for } 0 \leq t<\frac{1}{4}
$$

$$
\hat{\varphi}(t), \check{\varphi}(t), \tilde{\varphi}(t) \ll \frac{1+|\log (X / Z)|}{1+X / Z} \quad \text { for } t \geq 0
$$

$$
\hat{\varphi}(t), \check{\varphi}(t), \tilde{\varphi}(t) \ll\left(\frac{Z}{t}\right)\left(\frac{1}{t^{1 / 2}}+\frac{X}{t}\right) \quad \text { for } t \geq 1
$$

$$
\hat{\varphi}(t), \check{\varphi}(t), \tilde{\varphi}(t) \ll_{k}\left(\frac{Z}{t}\right)^{k}\left(\frac{1}{t^{1 / 2}}+\frac{X}{t}\right) \quad \text { for } t \geq \max (2 X, 1)
$$

Proof. The inequalities (2.11), (2.12), (2.13) can be proved exactly as (7.1), (7.2) and (7.3) in [DI82]. The last inequality (2.14) is an extension of (7.4) in [DI82], but we only claim it in the restricted range $t \geq \max (2 X, 1)$. On the one hand, we were unable to reconstruct the proof of (7.4) in [DI82] for the entire range $t \geq 1$; on the other hand, [DI82] only utilizes this inequality for $t \gg \max (X, Z)$ (cf. page 268 there, and note also that for $t \ll Z$ the bound (2.13) is stronger). For this reason we include a detailed proof of (2.14) in the case of $\check{\varphi}(t)$. For $\hat{\varphi}(t)$ and $\tilde{\varphi}(t)$ the proof is similar.

We may assume that $k=2 j+1$ is a positive odd integer. The Bessel differential equation

$$
x^{2} K_{2 i t}^{\prime \prime}(x)+x K_{2 i t}^{\prime}(x)=\left(x^{2}-4 t^{2}\right) K_{2 i t}(x)
$$

gives an identity 


$$
\check{\varphi}(t)=\left(D_{t} \varphi\right)^{\vee}(t)
$$

where

$$
D_{t} \varphi(x):=x\left(\frac{x \varphi(x)}{x^{2}-4 t^{2}}\right)^{\prime \prime}+x\left(\frac{\varphi(x)}{x^{2}-4 t^{2}}\right)^{\prime} .
$$

This transform $D_{t} \varphi$ is smooth and compactly supported in $(X, 2 X)$, and it is straightforward to check that

$$
\left\|\left(D_{t} \varphi\right)^{(i)}\right\|_{\infty} \ll_{i}(Z / t)^{2}(Z / X)^{i} \text { for } t \geq \max (2 X, 1) .
$$

By iterating (2.15) it follows that

$$
\check{\varphi}(t)=\left(D_{t}^{j} \varphi\right)^{\vee}(t),
$$

where $D_{t}^{j} \varphi$ is a smooth function, compactly supported in $(X, 2 X)$, satisfying

$$
\left\|\left(D_{t}^{j} \varphi\right)^{(i)}\right\|_{\infty} \ll_{j, i}(Z / t)^{2 j}(Z / X)^{i} \text { for } t \geq \max (2 X, 1) .
$$

We bound $\left(D_{t}^{j} \varphi\right)^{\vee}(t)$ by $(2.13)$ and obtain

$$
\check{\varphi}(t) \ll_{j}\left(\frac{Z}{t}\right)^{2 j+1}\left(\frac{1}{t^{1 / 2}}+\frac{X}{t}\right) \text { for } t \geq \max (2 X, 1) .
$$

Finally, we recall the large sieve inequalities from [DI82].

Theorem 3. Let $D$ be a positive integer, $M, K, T \geq 1$, and let $\left(a_{m}\right)_{m \sim M}$ be a sequence of complex numbers supported on $[M, 8 M]$. Then, for any $\varepsilon>0$,

$$
\begin{aligned}
& \sum_{\substack{k \equiv 0(2) \\
k \leq K}} \Gamma(k) \sum_{f \in \mathscr{B}_{k}^{h}(D)}\left|\sum_{m} a_{m} \sqrt{m} \rho_{f}(m)\right|^{2} \ll_{\varepsilon} M^{\varepsilon}\left(K^{2}+\frac{M}{D}\right) \sum_{m}\left|a_{m}\right|^{2} \\
& \sum_{\left|t_{j}\right| \leq T} \frac{1}{\operatorname{ch}\left(\pi t_{j}\right)}\left|\sum_{m} a_{m} \sqrt{m} \rho_{j}(m)\right|^{2} \ll_{\varepsilon} M^{\varepsilon}\left(T^{2}+\frac{M}{D}\right) \sum_{m}\left|a_{m}\right|^{2} \\
& \sum_{\mathfrak{a}} \int_{-T}^{T} \frac{1}{\operatorname{ch}(\pi t)}\left|\sum_{m} a_{m} \sqrt{m} \rho_{\mathfrak{a}}(m ; t)\right|^{2} d t \ll_{\varepsilon} M^{\varepsilon}\left(T^{2}+\frac{M}{D}\right) \sum_{m}\left|a_{m}\right|^{2} .
\end{aligned}
$$

\section{A large sieve inequality}

Theorem 4. Let $D, q, r$ be positive integers. For $M, N, C, Z \geq 1$, let $g(m, n ; c)$ be a smooth function compactly supported on 


$$
[M / 2,2 M] \times[N / 2,2 N] \times[C / 2,2 C]
$$

satisfying

$$
\frac{\partial^{i+j+k}}{\partial m^{i} \partial n^{j} \partial c^{k}} g(m, n ; c) \ll \ll_{i, j, k} \frac{Z^{i+j+k}}{M^{i} N^{j} C^{k}},
$$

and let $\left(a_{m}\right)_{m \in[M / 2,2 M]},\left(b_{n}\right)_{n \in[N / 2,2 N]}$ be two sequences of complex numbers satisfying

$$
\begin{aligned}
& a_{m} \neq 0 \Rightarrow q \mid m, \quad(q, m / q)=1, \\
& b_{n} \neq 0 \Rightarrow r \mid n, \quad(r, n / r)=1 .
\end{aligned}
$$

Then for any $\varepsilon>0$ one has, under Hypothesis $H_{\theta}$,

$$
\begin{aligned}
\Sigma^{ \pm}(M, N ; C) & :=\sum_{c \equiv 0(D)} \sum_{m} \sum_{n} a_{m} b_{n} \frac{S(m, \pm n ; c)}{c} g(m, n ; c) \\
& \ll_{\varepsilon}(q r D Z M N C)^{\varepsilon}(q r)^{\theta}\left(1+\frac{C}{\sqrt{M N}}\right)^{2 \theta} \\
& \times Z^{2}\left(Z^{3 / 2}+Z \frac{\sqrt{M N}}{C}+Z^{2 \theta} \frac{(q, D) M}{q D}\right)^{1 / 2} \\
& \times\left(Z^{3 / 2}+Z \frac{\sqrt{M N}}{C}+Z^{2 \theta} \frac{(r, D) N}{r D}\right)^{1 / 2}\|a\|_{2}\|b\|_{2} .
\end{aligned}
$$

Proof. This is a variant of Theorem 9 of [DI82]. We only treat the bound for $\Sigma^{+}(M, N ; C)$, the bound for $\Sigma^{-}(M, N ; C)$ being similar. We first proceed as in [DI82], and put the test function in a shape appropriate for the use of Kuznetsov's trace formula: We define $G$ by

$$
g\left(x_{1}, x_{2}, \frac{4 \pi \sqrt{x_{1} x_{2}}}{x}\right)=\iint_{\mathbb{R}^{2}} G\left(\xi_{1}, \xi_{2} ; x\right) e\left(\xi_{1} x_{1}+\xi_{2} x_{2}\right) d \xi_{1} d \xi_{2}
$$

so that by Fourier inversion one has

$$
G\left(\xi_{1}, \xi_{2} ; x\right)=\int_{M / 2}^{2 M} \int_{N / 2}^{2 N} g\left(x_{1}, x_{2}, \frac{4 \pi \sqrt{x_{1} x_{2}}}{x}\right) e\left(-\xi_{1} x_{1}-\xi_{2} x_{2}\right) d x_{1} d x_{2}
$$

and 


$$
\begin{aligned}
& \Sigma^{+}(M, N ; C) \\
& =\iint_{\mathbb{R}^{2}} \sum_{c \equiv 0(D)} \sum_{m} \sum_{n} a_{m} e\left(\xi_{1} m\right) b_{n} e\left(\xi_{2} n\right) \frac{S(m, n ; c)}{c} G\left(\xi_{1}, \xi_{2} ; \frac{4 \pi \sqrt{m n}}{c}\right) d \xi_{1} d \xi_{2} .
\end{aligned}
$$

Note that $G\left(\xi_{1}, \xi_{2} ; x\right)$ as a function of the $x$ variable is supported in the interval $[X, 16 X]$ with

$$
X:=\frac{\pi \sqrt{M N}}{C} .
$$

Let $p_{1}, p_{2}, k$ be 3 positive integers. We integrate (3.2) by parts $p_{1}$ times with respect to $x_{1}$ and $p_{2}$ times with respect to $x_{2}$, and differentiate it $k$ times with respect to $x$ getting

$$
\frac{\partial^{k}}{\partial x^{k}} G\left(\xi_{1}, \xi_{2} ; x\right) \ll_{p_{1}, p_{2}, k}\left(\frac{Z}{\left|\xi_{1}\right| M}\right)^{p_{1}}\left(\frac{Z}{\left|\xi_{2}\right| N}\right)^{p_{2}}\left(\frac{Z}{X}\right)^{k} M N .
$$

We postpone the integration over $\xi_{1}, \xi_{2}$ and the choice of $p_{1}, p_{2}$ to the end of section 3. Having these parameters fixed for the moment, we simplify the notation and set

$$
\varphi(x):=G\left(\xi_{1}, \xi_{2} ; x\right),
$$

and by slight abuse of notation we denote by $a_{m}$ and $b_{n}$ the complex numbers $a_{m} e\left(\xi_{1} m\right)$ and $b_{n} e\left(\xi_{2} n\right)$, respectively. This, of course, does not change the values of $\|a\|_{2},\|b\|_{2}$ or the support of these sequences. We apply Theorem 2, so that the integrand in (3.3) is the sum of three terms:

$$
T^{\text {Holo }}+T^{\text {Maass }}+T^{\text {Eisen }},
$$

where

$$
\begin{aligned}
T^{\text {Holo }} & :=4 \sum_{k \equiv 0} \tilde{\varphi}(2) \\
T^{\text {Maass }} & :=4 \sum_{j \geq 1} \frac{\hat{\varphi}\left(t_{j}\right)}{\operatorname{ch}\left(\pi t_{j}\right)}\left(\sum_{m \sim M} a_{m} \sqrt{m} \overline{\rho_{j}}(m)\right)\left(\sum_{n \sim N} b_{n} \sqrt{n} \rho_{j}(n)\right), \\
T^{\text {Eisen }} & :=\frac{1}{\pi} \sum_{\mathfrak{a}} \int_{-\infty}^{+\infty} \frac{\hat{\varphi}(t)}{\operatorname{ch}(\pi t)}\left(\sum_{m \sim M} a_{m} \sqrt{m} \overline{\rho_{f}}(m)\right)\left(\sum_{m \sim N} b_{n} \sqrt{n} \rho_{f}(n)\right),
\end{aligned}
$$

3.1. Contribution of the cuspidal spectrum. In this section we bound the contribution from the holomorphic and the Maass spectrum. The proof of the bound for $T^{\mathrm{Holo}}$ is 
similar to that of $T^{\text {Maass }}$, but sharper, so we only display the proof for $T^{\text {Maass }}$. By Cauchy-Schwarz we have

$$
\left|T^{\text {Maass }}\right|^{2} \ll\left(\sum_{j \geq 1} \frac{\left|\hat{\varphi}\left(t_{j}\right)\right|}{\operatorname{ch}\left(\pi t_{j}\right)}\left|\sum_{m \sim M} a_{m} \sqrt{m} \rho_{j}(m)\right|^{2}\right) \times\left(\sum_{j \geq 1} \frac{\left|\hat{\varphi}\left(t_{j}\right)\right|}{\operatorname{ch}\left(\pi t_{j}\right)}\left|\sum_{n \sim N} b_{n} \sqrt{n} \rho_{j}(n)\right|^{2}\right),
$$

so that it is sufficient to bound each factor separately. Our aim is to establish multiplicative properties of the coefficients $a_{m}, b_{n}$ in order to exploit the condition (3.1). Since the Hecke operators $T_{n}$ with $(n, D)=1$ are normal and commute with the Laplacian, one may choose an orthonormal basis $\left\{f_{j}\right\}$ of $\mathscr{B}(D)$ made of HeckeMaass eigenforms. More precisely, it follows from Atkin-Lehner theory that an orthonormal basis of Hecke-Maass eigenforms can be obtained as follows. Let $f$ be any Maass newform of level $D_{f}$ dividing $D$, then the complex vector space generated by $\left\{f_{d}(z):=f(d z), d \mid \frac{D}{D_{f}}\right\}$ is a $\tau\left(D / D_{f}\right)$-dimensional subspace of the space of cusp forms of level $D$ formed of Hecke eigenforms of the $T_{n},(n, D)=1$, with eigenvalues being the same as those of $f$, that is, $\lambda_{f}(n)$. By Gram-Schmidt there is an $L^{2}$-orthonormal basis $\left\{f_{(d)}(z), d \mid \frac{D}{D_{f}}\right\}$ of this subspace of the form

$$
f_{(d)}(z)=\sum_{d^{\prime} \mid D / D_{f}} \alpha_{d}\left(d^{\prime}\right) f\left(d^{\prime} z\right), \quad \alpha_{d}\left(d^{\prime}\right) \in \mathbb{C}
$$

(the $\alpha_{d}\left(d^{\prime}\right)$ depend also on $f$ but we suppress it from the notations). Now we form an orthonormal basis of Hecke-Maass cusp forms of level $D$ by the union of the $\left\{f_{(d)}(z), d \mid \frac{D}{D_{f}}\right\}$ for $f$ ranging over the $L^{2}$-normalized cuspidal newforms of level dividing $D$. Let us fix one of these basis elements $f_{(d)}$ for a moment. We have for any $m \geq 1$,

$$
\sqrt{m} \rho_{f_{(d)}}(m)=\sum_{d^{\prime} \mid D} \alpha_{d}\left(d^{\prime}\right) \sqrt{m} \rho_{f_{d^{\prime}}}(m)=\sum_{d^{\prime} \mid(D, m)} \sqrt{d^{\prime}} \alpha_{d}\left(d^{\prime}\right) \sqrt{m / d^{\prime}} \rho_{f}\left(m / d^{\prime}\right)
$$

where, in order to simplify notation, we made the convention that $\alpha_{d}\left(d^{\prime}\right)=0$ if $d$ or $d^{\prime}$ does not divide $D / D_{f}$. We suppose now that $m=q m^{\prime}$ with $\left(q, m^{\prime}\right)=1$. Setting $q^{\prime}=(q, D)$, we obtain by $(2.7)$ that

$$
\begin{aligned}
\sqrt{m} \rho_{f_{(d)}}(m) & =\sum_{\substack{d_{1}\left|\left(D, m^{\prime}\right) \\
d_{2}\right| q^{\prime}}} \sqrt{d_{1} d_{2}} \alpha_{d}\left(d_{1} d_{2}\right) \sqrt{\frac{q m^{\prime}}{d_{2} d_{1}}} \rho_{f}\left(\frac{q m^{\prime}}{d_{2} d_{1}}\right) \\
& =\sum_{\substack{d_{1}\left|\left(D, m^{\prime}\right) \\
d_{2}\right| q^{\prime}}} \sqrt{d_{1} d_{2}} \alpha_{d}\left(d_{1} d_{2}\right) \lambda_{f}\left(\frac{q}{d_{2}}\right) \sqrt{\frac{m^{\prime}}{d_{1}}} \rho_{f}\left(\frac{m^{\prime}}{d_{1}}\right)
\end{aligned}
$$


since $f$ is a Hecke eigenform for all Hecke operators. For the same reason, we have the identity

$$
\lambda_{f}\left(\frac{q}{d_{2}}\right)=\lambda_{f}\left(\frac{q}{q^{\prime}} \frac{q^{\prime}}{d_{2}}\right)=\sum_{d_{3} \mid\left(q / q^{\prime}, q^{\prime} / d_{2}\right)} \chi_{f}\left(d_{3}\right) \mu\left(d_{3}\right) \lambda_{f}\left(\frac{q}{q^{\prime} d_{3}}\right) \lambda_{f}\left(\frac{q^{\prime}}{d_{2} d_{3}}\right) .
$$

Since $\left(q^{\prime}, m^{\prime}\right)=1$, we conclude that

$$
\begin{aligned}
& \sqrt{m} \rho_{f_{(d)}}(m) \\
& \quad=\sum_{d_{3} \mid\left(q / q^{\prime}, q^{\prime}\right)} \chi_{f}\left(d_{3}\right) \mu\left(d_{3}\right) \lambda_{f}\left(\frac{q}{q^{\prime} d_{3}}\right) \sum_{\substack{d_{1}\left|\left(D, m^{\prime}\right) \\
d_{2}\right| q^{\prime} / d_{3}}} \sqrt{d_{1} d_{2}} \alpha_{d}\left(d_{1} d_{2}\right) \sqrt{\frac{q^{\prime} m^{\prime}}{d_{3} d_{2} d_{1}}} \rho_{f}\left(\frac{q^{\prime} m^{\prime}}{d_{3} d_{2} d_{1}}\right) \\
& \quad=\sum_{d_{3} \mid\left(q / q^{\prime}, q^{\prime}\right)} \chi_{f}\left(d_{3}\right) \mu\left(d_{3}\right) \lambda_{f}\left(\frac{q}{q^{\prime} d_{3}}\right) \sum_{d^{\prime} \mid\left(D, q^{\prime} m^{\prime} / d_{3}\right)} \sqrt{d^{\prime}} \alpha_{d}\left(d^{\prime}\right) \sqrt{\frac{q^{\prime} m^{\prime}}{d_{3} d^{\prime}}} \rho_{f}\left(\frac{q^{\prime} m^{\prime}}{d_{3} d^{\prime}}\right) \\
& \quad=\sum_{d_{3} \mid\left(q / q^{\prime}, q^{\prime}\right)} \chi_{f}\left(d_{3}\right) \mu\left(d_{3}\right) \lambda_{f}\left(\frac{q}{q^{\prime} d_{3}}\right) \sqrt{\frac{q^{\prime} m^{\prime}}{d_{3}}} \rho_{f_{(d)}}\left(\frac{q^{\prime} m^{\prime}}{d_{3}}\right) .
\end{aligned}
$$

Hence it follows from (3.1) and (2.4) that for $f_{j}$ an element of Hecke eigenbasis described above (and by writing $d$ for $\frac{q^{\prime}}{d_{3}}$ ),

$$
\left|\sum_{m} a_{m} \sqrt{m} \rho_{f_{j}}(m)\right|^{2} \leq \tau(q)^{2} q^{2 \theta} \sum_{d \mid(q, D)}\left|\sum_{\left(m^{\prime}, q\right)=1} a_{q m^{\prime}} \sqrt{d m^{\prime}} \rho_{f_{j}}\left(d m^{\prime}\right)\right|^{2}
$$

and therefore

$$
\sum_{j \geq 1} \frac{\left|\hat{\varphi}\left(t_{j}\right)\right|}{\operatorname{ch}\left(\pi t_{j}\right)}\left|\sum_{m} a_{m} \sqrt{m} \rho_{j}(m)\right|^{2} \leq \tau(q)^{2} q^{2 \theta} \sum_{d \mid(q, D)} \sum_{j \geq 1} \frac{\left|\hat{\varphi}\left(t_{j}\right)\right|}{\operatorname{ch}\left(\pi t_{j}\right)}\left|\sum_{m^{\prime} \sim M / q} a_{q m^{\prime}} \sqrt{d m^{\prime}} \rho_{j}\left(d m^{\prime}\right)\right|^{2} .
$$

To estimate the $j$-sum we set

$$
T_{0}:=\max \left(16 X,(Z X)^{1 / 2}, Z^{2 / 3}\right),
$$

where $X$ is given by (3.4), and we split the sum as

$$
\sum_{j \geq 1} \frac{\left|\hat{\varphi}\left(t_{j}\right)\right|}{\operatorname{ch}\left(\pi t_{j}\right)}\left|\sum_{m^{\prime} \sim M / q} a_{q m^{\prime}} \sqrt{d m^{\prime}} \rho_{j}\left(d m^{\prime}\right)\right|^{2}=\sum_{\left|t_{j}\right| \leq 1} \cdots+\sum_{1<\left|t_{j}\right| \leq T_{0}} \cdots+\sum_{T} \sum_{T<\left|t_{j}\right| \leq 2 T} \cdots
$$

where $T$ runs through the numbers of the form $2^{v} T_{0}, v \in \mathbb{N}_{0}$. 
By the large sieve inequality (2.17) combined with (3.5), (2.11) and (2.12), we obtain

$$
\sum_{\left|t_{j}\right| \leq 1} \cdots \ll_{\varepsilon, p_{1}, p_{2}}((1+X) Z)^{2 \varepsilon}\left(\frac{Z}{\left|\xi_{1}\right| M}\right)^{p_{1}}\left(\frac{Z}{\left|\xi_{2}\right| N}\right)^{p_{2}} M N\left(1+\frac{Z}{X}\right)^{2 \theta}\left(1+\frac{d M}{q D}\right)\|a\|_{2}^{2} .
$$

Similarly, a combination of (2.17), (3.5) and (2.12) shows that

$$
\begin{aligned}
\sum_{1<\left|t_{j}\right| \leq T_{0}} \cdots & \ll_{\varepsilon, p_{1}, p_{2}}((1+X) Z)^{2 \varepsilon}\left(\frac{Z}{\left|\xi_{1}\right| M}\right)^{p_{1}}\left(\frac{Z}{\left|\xi_{2}\right| N}\right)^{p_{2}} M N\left(\frac{Z}{Z+X}\right)\left(T_{0}^{2}+\frac{d M}{q D}\right)\|a\|_{2}^{2} \\
& \ll_{\varepsilon, p_{1}, p_{2}}((1+X) Z)^{2 \varepsilon}\left(\frac{Z}{\left|\xi_{1}\right| M}\right)^{p_{1}}\left(\frac{Z}{\left|\xi_{2}\right| N}\right)^{p_{2}} M N\left(Z^{4 / 3}+Z X+\frac{d M}{q D}\right)\|a\|_{2}^{2} .
\end{aligned}
$$

For each $T=2^{v} T_{0}$ such that $T \leq Z^{1+\varepsilon}$ we can combine (2.17), (3.5) and (2.13) to see that

$$
\begin{gathered}
\sum_{T<\left|t_{j}\right| \leq 2 T} \cdots \ll_{\varepsilon, p_{1}, p_{2}} Z^{\varepsilon}\left(\frac{Z}{\left|\xi_{1}\right| M}\right)^{p_{1}}\left(\frac{Z}{\left|\xi_{2}\right| N}\right)^{p_{2}} M N\left(\frac{Z}{T}\right)\left(\frac{1}{T^{1 / 2}}+\frac{X}{T}\right)\left(T^{2}+\frac{d M}{q D}\right)\|a\|_{2}^{2} \\
\ll_{\varepsilon, p_{1}, p_{2}} Z^{2 \varepsilon}\left(\frac{Z}{\left|\xi_{1}\right| M}\right)^{p_{1}}\left(\frac{Z}{\left|\xi_{2}\right| N}\right)^{p_{2}} M N\left(Z^{3 / 2}+Z X+\frac{d M}{q D}\right)\|a\|_{2}^{2} .
\end{gathered}
$$

The contribution of each $T=2^{v} T_{0}$ such that $T>Z^{1+\varepsilon}$ is negligible as follows from (2.17), (3.5) and (2.14) with $k=10 / \varepsilon$. In fact, we have $Z<T^{1-\varepsilon / 2}$, therefore

$$
\begin{aligned}
\sum_{T<\left|t_{j}\right| \leq 2 T} \cdots & \ll_{\varepsilon, p_{1}, p_{2}} T^{\varepsilon}\left(\frac{Z}{\left|\xi_{1}\right| M}\right)^{p_{1}}\left(\frac{Z}{\left|\xi_{2}\right| N}\right)^{p_{2}} M N\left(\frac{Z}{T}\right)^{10 / \varepsilon}\left(\frac{1}{T^{1 / 2}}+\frac{X}{T}\right)\left(T^{2}+\frac{d M}{q D}\right)\|a\|_{2}^{2} \\
& \ll \varepsilon_{\varepsilon, p_{1}, p_{2}}\left(\frac{Z}{\left|\xi_{1}\right| M}\right)^{p_{1}}\left(\frac{Z}{\left|\xi_{2}\right| N}\right)^{p_{2}} M N T^{-2}\left(1+\frac{d M}{q D}\right)\|a\|_{2}^{2} .
\end{aligned}
$$

By summing over all $T \geq T_{0}$ and using also (3.8) we infer that

$$
\begin{aligned}
& \sum_{j \geq 1} \frac{\left|\hat{\varphi}\left(t_{j}\right)\right|}{\operatorname{ch}\left(\pi t_{j}\right)}\left|\sum_{m} a_{m} \sqrt{m} \rho_{j}(m)\right|^{2} \\
& \ll_{\varepsilon, p_{1}, p_{2}}((1+X) Z q)^{3 \varepsilon}\left(\frac{Z}{\left|\xi_{1}\right| M}\right)^{p_{1}}\left(\frac{Z}{\left|\xi_{2}\right| N}\right)^{p_{2}} M N \\
& \times q^{2 \theta}\left(1+\frac{1}{X}\right)^{2 \theta}\left(Z^{3 / 2}+Z X+Z^{2 \theta} \frac{(q, D) M}{q D}\right)\|a\|_{2}^{2} .
\end{aligned}
$$


We have a similar bound for the second factor in (3.7). Therefore we obtain, for any $\varepsilon>0$,

$$
\begin{aligned}
T^{\text {Maass }} \ll_{\varepsilon, p_{1}, p_{2}}((1+X) Z q r)^{\varepsilon}\left(\frac{Z}{\left|\xi_{1}\right| M}\right)^{p_{1}}\left(\frac{Z}{\left|\xi_{2}\right| N}\right)^{p_{2}} M N \\
\times(q r)^{\theta}\left(1+\frac{1}{X}\right)^{2 \theta}\left(Z^{3 / 2}+Z X+Z^{2 \theta} \frac{(q, D) M}{q D}\right)^{1 / 2} \\
\times\left(Z^{3 / 2}+Z X+Z^{2 \theta} \frac{(r, D) N}{r D}\right)^{1 / 2}\|a\|_{2}\|b\|_{2} .
\end{aligned}
$$

The contribution of holomorphic forms is treated similarly using (2.16); however, since the Ramanujan-Petersson conjecture holds true for holomorphic forms, one obtains the stronger bound

$$
\begin{aligned}
& T^{\text {Holo }} \ll_{\varepsilon, p_{1}, p_{2}}((1+X) Z q r)^{\varepsilon}\left(\frac{Z}{\left|\xi_{1}\right| M}\right)^{p_{1}}\left(\frac{Z}{\left|\xi_{2}\right| N}\right)^{p_{2}} M N \\
& \quad \times\left(Z^{3 / 2}+Z X+\frac{(q, D) M}{q D}\right)^{1 / 2}\left(Z^{3 / 2}+Z X+\frac{(r, D) N}{r D}\right)^{1 / 2}\|a\|_{2}\|b\|_{2} .
\end{aligned}
$$

3.2. Contribution of the Eisenstein spectrum. We now evaluate $T^{\text {Eisen }}$ in (3.6). By Cauchy-Schwarz one has

$$
\left|T^{\text {Eisen }}\right|^{2} \ll\left(\int_{\mathbb{R}} \frac{|\hat{\varphi}(t)|}{\operatorname{ch}(\pi t)} \sum_{\mathfrak{a}}\left|\sum_{m} a_{m} \sqrt{m} \rho_{\mathfrak{a}}(m ; t)\right|^{2} d t\right) \times\left(\int_{\mathbb{R}} \frac{|\hat{\varphi}(t)|}{\operatorname{ch}(\pi t)} \sum_{\mathfrak{a}}\left|\sum_{n} b_{n} \sqrt{n} \rho_{\mathfrak{a}}(n)\right|^{2} d t\right),
$$

and it is sufficient to bound each factor separately. We wish to imitate the argument given above, but a slight difficulty occurs as the Eisenstein series $E_{\mathfrak{a}}(z, s)$ are not Hecke eigenforms in general. The problem of diagonalizing Hecke operators on the space of Eisenstein series was dealt with by Rankin [Ra90, Ra93, Ra92, Ra94]. However, we proceed (as in [M04a, HM04a]) by computing directly the Fourier coefficients of the Eisenstein series. Recall (see [DI82, Lemma 2.3]) that the cusps $\{\mathfrak{a}\}$ of $\Gamma_{0}(D)$ are uniquely represented by the rationals

$$
\left\{\frac{u}{w}: w \mid D, u \in \mathscr{U}_{w}\right\}
$$

where, for each $w \mid D, \mathscr{U}_{w}$ is a set of integers coprime with $w$ representing each reduced residue class modulo $\tilde{w}:=(w, D / w)$ exactly once. In the half-plane $\Im t<0$ we have for $m \neq 0$ (see [DI82, (1.17) and p. 247]), 


$$
\sqrt{|m|} \rho_{\mathfrak{a}}(m, t)=\frac{\pi^{1 / 2+i t}|m|^{i t}}{\Gamma\left(\frac{1}{2}+i t\right)}\left(\frac{(w, D / w)}{w D}\right)^{1 / 2+i t} \sum_{\substack{(\gamma, D / w)=1 \\ \gamma^{1+2 i t}}} \sum_{\substack{\delta(\bmod \gamma w),(\delta, \gamma w)=1 \\ \delta \gamma \equiv u(\bmod \tilde{w})}} e\left(-m \frac{\delta}{\gamma w}\right)
$$

with analytic continuation to $\Im t=0$. The congruence condition on $\delta$ can be analyzed by means of multiplicative characters modulo $\tilde{w}$ :

$$
\begin{aligned}
& \sum_{\substack{(\gamma, D / w)=1 \\
\gamma^{1+2 i t}}} \sum_{\substack{\delta(\bmod \gamma w),(\delta, \gamma w)=1 \\
\delta \gamma \equiv u(\bmod \tilde{w})}} e\left(-m \frac{\delta}{\gamma w}\right) \\
& =\frac{1}{\varphi(\tilde{w})} \sum_{\psi \bmod \tilde{w}} \bar{\psi}(-u) \sum_{(\gamma, D / w)=1} \frac{\psi(\gamma)}{\gamma^{1+2 i t}} G_{\psi}(m ; \gamma w),
\end{aligned}
$$

where

$$
G_{\psi}(m, q):=\sum_{b(\bmod q)}^{*} \psi(b) e\left(\frac{b m}{q}\right)
$$

is the Gauss sum. Note that we may replace $\psi$ by its underlying primitive character, since we only sum over $b$ coprime with $q$. For brevity we write

$$
S_{\psi}(m ; w):=\sum_{(\gamma, D / w)=1} \frac{\psi(\gamma)}{\gamma^{1+2 i t}} G_{\psi}(m ; \gamma w)
$$

so that

$$
\begin{aligned}
\sum_{\mathfrak{a}}\left|\sum_{m} a_{m} \sqrt{m} \rho_{\mathfrak{a}}(m, t)\right|^{2} & \\
& =\frac{\pi}{\left|\Gamma\left(\frac{1}{2}+i t\right)\right|^{2}} \sum_{w \mid D} \frac{\tilde{w}}{w D \varphi^{2}(\tilde{w})} \sum_{u \in \mathscr{U}_{w}}\left|\sum_{\psi \bmod \tilde{w}} \bar{\psi}(-u) \sum_{m} a_{m} m^{i t} S_{\psi}(m ; w)\right|^{2} .
\end{aligned}
$$

By Parseval it follows that

$$
\sum_{\mathfrak{a}}\left|\sum_{m} a_{m} \sqrt{m} \rho_{\mathfrak{a}}(m, t)\right|^{2}=\frac{\pi}{\left|\Gamma\left(\frac{1}{2}+i t\right)\right|^{2}} \sum_{w \mid D} \frac{\tilde{w}}{w D \varphi(\tilde{w})} \sum_{\psi \bmod \tilde{w}}\left|\sum_{m} a_{m} m^{i t} S_{\psi}(m ; w)\right|^{2} .
$$

In the following it will be useful to perform the summation over the primitive characters underlying the $\psi$ 's. For each character $\psi \bmod \tilde{w}$, we record by $w^{*}$ its conductor and by $\psi^{*}$ its underlying primitive character, so that 


$$
\begin{aligned}
\sum_{\mathfrak{a}}\left|\sum_{m} a_{m} \sqrt{m} \rho_{\mathfrak{a}}(m, t)\right|^{2} & \\
& =\frac{\pi}{\left|\Gamma\left(\frac{1}{2}+i t\right)\right|^{2}} \sum_{w^{* 2}\left|D \psi^{*} \bmod w^{*} w^{*}\right| w \mid D / w^{*}} \frac{\tilde{w}}{w D \varphi(\tilde{w})}\left|\sum_{m} a_{m} m^{i t} S_{\psi^{*}}(m ; w)\right|^{2},
\end{aligned}
$$

where the $\psi^{*}$-sum is over primitive characters only. To compute $S_{\psi^{*}}(m ; w)$, we decompose $w$ as

$$
w=w^{*} w^{\prime} w^{\prime \prime}, \quad w^{\prime} \mid\left(w^{*}\right)^{\infty}, \quad\left(w^{\prime \prime}, w^{*}\right)=1 .
$$

Our aim is now to establish (3.13) below, i.e. for $m=q m^{\prime}$ with $\left(q, m^{\prime}\right)=1$ we want to express $S_{\psi^{*}}\left(q m^{\prime}, w\right)$ in terms of $S_{\psi^{*}}\left(d m^{\prime}, *\right)$ with $d \mid(q, D)$. Let us first note that according to our decomposition of $w$ the Gauss sum factors as

$$
\begin{aligned}
G_{\psi^{*}}(m ; \gamma w) & =\psi^{*}\left(\gamma w^{\prime \prime}\right) G_{\psi^{*}}\left(m ; w^{*} w^{\prime}\right) r\left(m ; \gamma w^{\prime \prime}\right) \\
& =\delta_{w^{\prime} \mid m} w^{\prime} \psi^{*}\left(\gamma w^{\prime \prime}\right) G_{\psi^{*}}\left(m / w^{\prime} ; w^{*}\right) r\left(m ; \gamma w^{\prime \prime}\right),
\end{aligned}
$$

where $r(m ; q):=G_{1}(m ; q)$ is the Ramanujan sum and $\delta_{w^{\prime} \mid m}=1$ if $w^{\prime} \mid m$ and else it vanishes. With this notation,

$$
\begin{aligned}
S_{\psi^{*}}(m ; w)= & \frac{\delta_{w^{\prime} \mid m} w^{\prime} \overline{\psi^{*}}\left(m / w^{\prime}\right) \psi^{*}\left(w^{\prime \prime}\right) G_{\psi^{*}}\left(1 ; w^{*}\right)}{L^{(D)}\left(\psi^{* 2}, 1+2 i t\right)} \\
& \times\left(\sum_{\substack{\gamma \mid D^{\infty} \\
(\gamma, D / w)=1}} \frac{\psi^{* 2}(\gamma)}{\gamma^{1+2 i t}} r\left(m ; \gamma w^{\prime \prime}\right)\right)\left(\sum_{\substack{a \mid m \\
(a, D)=1}} \frac{\psi^{* 2}(a)}{a^{2 i t}}\right) \\
= & \frac{\delta_{w^{\prime} \mid m} w^{\prime} \overline{\psi^{*}}\left(m / w^{\prime}\right) \psi^{*}\left(w^{\prime \prime}\right) G_{\psi^{*}}\left(1 ; w^{*}\right)}{L^{(D)}\left(\psi^{* 2}, 1+2 i t\right)} R_{\psi^{*}}\left(m ; w^{\prime \prime}\right) \eta_{\psi^{*}}(m),
\end{aligned}
$$

say, where the superscript $(D)$ indicates that the local factors at the primes dividing $D$ have been removed. We consider the $\gamma$-sum

$$
R_{\psi^{*}}\left(m ; w^{\prime \prime}\right)=\sum_{\substack{\gamma \mid D^{\infty} \\(\gamma, D / w)=1}} \frac{\psi^{* 2}(\gamma)}{\gamma^{1+2 i t}} r\left(m ; \gamma w^{\prime \prime}\right) .
$$

Since $\left(\gamma, w^{*}\right)=(\gamma, D / w)=1$, it follows that in fact $\gamma \mid\left(w^{\prime \prime}\right)^{\infty}$ (justifying our notation), and one has 


$$
R_{\psi^{*}}\left(m ; w^{\prime \prime}\right)=\prod_{\substack{p^{\alpha}\left\|w^{\prime \prime} \\ p^{\alpha}\right\| D}}\left(\sum_{\beta \geq 0} \frac{\psi^{* 2}\left(p^{\beta}\right)}{p^{\beta(1+2 i t)}} r\left(p^{v_{p}(m)} ; p^{\alpha+\beta}\right)\right)
$$

We suppose now that $m$ is of the form $m=q m^{\prime}$ with $\left(q, m^{\prime}\right)=1$, and we factor $w^{\prime}$, $w^{\prime \prime}$ (recall that they are coprime) as

$$
w^{\prime}=w_{q}^{\prime} w^{\prime(q)}, w^{\prime \prime}=w_{q}^{\prime \prime} w^{\prime \prime(q)}, \quad \text { where } w_{q}^{\prime}, w_{q}^{\prime \prime} \mid q^{\infty} \text { and }\left(w^{\prime(q)} w^{\prime \prime(q)}, q\right)=1 .
$$

Moreover, since $\left(q, m^{\prime}\right)=1$ and $w^{\prime} \mid q m^{\prime}$, it follows that $w_{q}^{\prime}=\left(w^{\prime}, q\right)$. With these notations we find that

$$
\begin{aligned}
S_{\psi^{*}}\left(q m^{\prime} ; w\right) & =\delta_{w_{q}^{\prime} \mid q} w_{q}^{\prime} \overline{\psi^{*}}\left(\frac{q}{w_{q}^{\prime}}\right) \psi^{*}\left(w_{q}^{\prime \prime}\right) R_{\psi^{*}}\left(q ; w_{q}^{\prime \prime}\right) \eta_{\psi^{*}}(q) \\
& \times \delta_{w^{\prime(q)} \mid m^{\prime}} w^{\prime(q)} \overline{\psi^{*}}\left(\frac{m^{\prime}}{w^{\prime(q)}}\right) \psi^{*}\left(w^{\prime \prime(q)}\right) \frac{G_{\psi^{*}}\left(1 ; w^{*}\right)}{L^{(D)}\left(\psi^{* 2}, 1+2 i t\right)} R_{\psi^{*}}\left(m^{\prime} ; w^{\prime \prime(q)}\right) \eta_{\psi^{*}}\left(m^{\prime}\right) .
\end{aligned}
$$

Setting

$$
v_{q}:=\left(\frac{w^{\prime \prime}}{\left(w^{\prime \prime}, 2\right)}, q\right) \text { and } \quad \tilde{v}_{q}:=\prod_{\substack{p^{\alpha} \| w_{q}^{\prime \prime} \\ \alpha \leq v_{p}(q)+1}} p^{\alpha}
$$

an explicit calculation shows that $R_{\psi^{*}}\left(v_{q} ; \tilde{v}_{q}\right)$ is nonzero and in fact

$$
\left|\frac{R_{\psi^{*}}\left(q ; w_{q}^{\prime \prime}\right)}{R_{\psi^{*}}\left(v_{q} ; \tilde{v}_{q}\right)}\right| \leq \prod_{p \mid(q, D)}\left(v_{p}(q)+1\right) \frac{1+\frac{1}{p}}{\kappa(p)} \leq 3 \tau(D) \tau(q),
$$

where

$$
\kappa(p):= \begin{cases}\frac{1}{2}, & p=2 \\ 1-\frac{2}{p}, & p>2\end{cases}
$$

Note also that $w_{q}^{\prime} v_{q} \mid(q, D)$ and $\tilde{v}_{q} \mid w_{q}^{\prime \prime}$. In particular, $\left(w_{q}^{\prime} v_{q}, m^{\prime}\right)=1$ and $\eta_{\psi^{*}}\left(w_{q}^{\prime} v_{q}\right)=1$, therefore by the above formula

$$
S_{\psi^{*}}\left(q m^{\prime} ; w\right)=\delta_{w_{q}^{\prime} \mid q} \overline{\psi^{*}}\left(\frac{q}{w_{q}^{\prime} v_{q}}\right) \psi^{*}\left(\frac{w_{q}^{\prime \prime}}{\tilde{v}_{q}}\right) \frac{R_{\psi^{*}}\left(q ; w_{q}^{\prime \prime}\right)}{R_{\psi^{*}}\left(v_{q} ; \tilde{v}_{q}\right)} \eta_{\psi^{*}}(q) S_{\psi^{*}}\left(w_{q}^{\prime} v_{q} m^{\prime} ; w^{*} w^{\prime} \tilde{v}_{q} w^{\prime \prime(q)}\right) .
$$

Combining (3.12) with (3.13) and noting that $\left|\eta_{\psi^{*}}(q)\right| \leq \tau(q)$ we obtain by a trivial estimation

$$
\left|\sum_{m} a_{m} m^{i t} S_{\psi}(m ; w)\right|^{2} \leq 9 \tau(D)^{2} \tau(q)^{4} \sum_{d \mid(q, D)}\left|\sum_{\left(m^{\prime}, q\right)=1} a_{q m^{\prime}}\left(m^{\prime}\right)^{i t} S_{\psi}\left(d m^{\prime} ; w^{*} w^{\prime} \tilde{v}_{q} w^{\prime \prime(q)}\right)\right|^{2} .
$$


Given $w^{*}$ such that $w^{* 2} \mid D$ and given $w$ such that $w^{*}|w| \frac{D}{w^{*}}$, the number $w_{1}:=$ $w^{*} w^{\prime} \tilde{v}_{q} w^{\prime \prime(q)}$ also satisfies $w^{*}\left|w_{1}\right| w \mid \frac{D}{w^{*}}$. Moreover, if we set $\tilde{w}_{1}:=\left(w_{1}, D / w_{1}\right)$, one has

$$
\frac{\tilde{w}}{\varphi(\tilde{w})} \leq \tau(D) \frac{\tilde{w}_{1}}{\varphi\left(\tilde{w}_{1}\right)},
$$

so we deduce from this discussion and from (3.11) that

$$
\begin{aligned}
\sum_{\mathfrak{a}}\left|\sum_{m} a_{m} \sqrt{m} \rho_{\mathfrak{a}}(m, t)\right|^{2} & \leq 9 \tau(D)^{3} \tau(q)^{4} \sum_{d \mid(q, D)} \frac{\pi}{\left|\Gamma\left(\frac{1}{2}+i t\right)\right|^{2}} \\
& \times \sum_{w^{* 2} \mid D} \sum_{\psi^{*}} \bmod \sum_{w^{*}} \sum_{w^{*}\left|w_{1}\right| D^{\prime}} \frac{\tilde{w}_{1}}{w_{1} D \varphi\left(\tilde{w}_{1}\right)}\left|\sum_{\left(m^{\prime}, q\right)=1} a_{q m^{\prime}}\left(m^{\prime}\right)^{i t} S_{\psi}\left(d m^{\prime} ; w_{1}\right)\right|^{2} \\
& =9 \tau(D)^{3} \tau(q)^{4} \sum_{d \mid(q, D)} \sum_{\mathfrak{a}}\left|\sum_{\left(m^{\prime}, q\right)=1} a_{q m^{\prime}} \sqrt{d m^{\prime}} \rho_{\mathfrak{a}}\left(d m^{\prime}, t\right)\right|^{2}
\end{aligned}
$$

We are now in a similar situation as in (3.8). Applying the large sieve inequality (2.18) we obtain analogously the bound

$$
\begin{aligned}
& T^{\text {Eisen }} \ll_{\varepsilon, p_{1}, p_{2}}((1+X) D Z q r)^{\varepsilon}\left(\frac{Z}{\left|\xi_{1}\right| M}\right)^{p_{1}}\left(\frac{Z}{\left|\xi_{2}\right| N}\right)^{p_{2}} M N \\
& \quad \times\left(Z^{3 / 2}+Z X+\frac{(q, D) M}{q D}\right)^{1 / 2}\left(Z^{3 / 2}+Z X+\frac{(r, D) N}{r D}\right)^{1 / 2}\|a\|_{2}\|b\|_{2}
\end{aligned}
$$

for any $\varepsilon>0$.

Collecting (3.4), (3.9), (3.10), (3.14), and integrating over the $\xi_{1}, \xi_{2}$ variables (with $p_{1}=0$ if $\left|\xi_{1}\right| \leq Z / M$, and $p_{1}>1$ if $\left|\xi_{1}\right|>Z / M$ and similarly for $\left.\left(\xi_{2}, p_{2}\right)\right)$ we conclude the proof of Theorem 4.

\section{A shifted convolution problem}

Let $q, l_{1}, l_{2}$ be positive integers, and $g$ be a cuspidal newform of level $D$ and nebentypus $\chi_{g}$ and Hecke eigenvalues $\lambda_{g}(n), n \geq 1$. Let $F(x, y)$ be a smooth function supported on $[X / 2,2 X] \times[Y / 2,2 Y]$ which satisfies

$$
\frac{\partial^{i+j}}{\partial x^{i} \partial y^{j}} F(x, y) \ll \frac{Z^{i+j}}{X^{i} Y^{j}}
$$

for some $X, Y, Z \geq 1$ and for all $i, j \geq 0$, the implied constant depending only on $i, j$. 
In this section we estimate, for positive integers $l_{1}, l_{2}, q$, the following average of shifted convolution sums:

$$
\mathscr{D}\left(g, l_{1}, l_{2}, q\right):=\sum_{h \neq 0} \phi(q h) \sum_{l_{1} m-l_{2} n=q h} \overline{\lambda_{g}}(m) \lambda_{g}(n) F\left(l_{1} m, l_{2} n\right)
$$

where by symmetry we may assume that $Y \geq X$ and $\phi(q h)$ is a redundancy factor (borrowed from [DFI94a] to ease the forthcoming computations) arising from a smooth even function $\phi$ such that $\phi_{[-2 Y, 2 Y]} \equiv 1$, supp $\phi \subset[-4 Y, 4 Y]$ and $\phi^{(i)}(x) \ll_{i} Y^{-i}$. Our analysis follows closely Sections 4.1-5.2 of [HM04a], but we also make use of the essential ingredients of $[\mathrm{B} 104 \mathrm{~b}]$, namely the square mean bound for shifted convolution sums taken from [J96] and the spectral large sieve of [DI82] in our improved form of Theorem 4. The proof of Proposition 2.4 of [HM04a] yields the following uniform estimate for exponential sums associated with $g$ :

$$
S_{g}(\alpha, x):=\sum_{n \leq x} \lambda_{g}(n) e(n \alpha) \ll_{\varepsilon}\left(D \mu_{g} x\right)^{\varepsilon} \omega_{g} D^{1 / 2} \mu_{g}^{1 / 2} x^{1 / 2}
$$

where

$$
\omega_{g}:=\frac{\|g\|_{\infty}}{\|g\|_{2}} .
$$

In the same proof we also demonstrated by an elementary argument that

$$
\omega_{g} \ll_{\varepsilon}\left(D \mu_{g}\right)^{\varepsilon} D^{1 / 2} \mu_{g}^{3 / 2}
$$

With this bound at hand we derive the following square mean bound for shifted convolution sums, a variant of Lemma 3 in [J96] (cf. Lemma 3.2 in [B104b]): for $l_{1}, l_{2} \in \mathbb{Z}$ and $x, y \geq 1$ we have by Rankin-Selberg theory

$$
\begin{aligned}
\sum_{h^{\prime} \in \mathbb{Z}}\left|\sum_{\substack{m \leq x, n \leq y \\
l_{1} m \pm l_{2} n=h^{\prime}}} \overline{\lambda_{g}}(m) \lambda_{g}(n)\right|^{2} & =\int_{0}^{1}\left|S_{g}\left(-l_{1} \alpha, x\right) S_{g}\left( \pm l_{2} \alpha, y\right)\right|^{2} d \alpha \\
& \ll_{\varepsilon}\left(D \mu_{g} x\right)^{\varepsilon} \omega_{g}^{2} D \mu_{g} x \int_{0}^{1}\left|S_{g}\left( \pm l_{2} \alpha, y\right)\right|^{2} d \alpha \\
& =\left(D \mu_{g} x\right)^{\varepsilon} \omega_{g}^{2} D \mu_{g} x \sum_{n \leq y}\left|\lambda_{g}(n)\right|^{2} \\
& \ll_{\varepsilon}\left(D \mu_{g} x y\right)^{2 \varepsilon} \omega_{g}^{2} D \mu_{g} x y
\end{aligned}
$$

the implied constant depending on $\varepsilon$ alone. Our goal is 
Theorem 5. Assume Hypothesis $H_{\theta}$. Set

$$
T:=q D \mu_{g} l_{1} l_{2} X Y Z
$$

and assume (by symmetry) that $Y \geq X$. Then

$$
\mathscr{D}\left(g, l_{1}, l_{2}, q\right) \ll_{\varepsilon} T^{\varepsilon} \omega_{g} D \mu_{g}^{63 / 4+3 \theta} Z^{25 / 4+\theta} q^{\theta-1 / 2} Y\left(\left(\frac{Y}{X}\right)^{1 / 2}+\frac{\left(q, D l_{1} l_{2}\right)}{q D l_{1} l_{2}} Y\right)^{1 / 2}
$$

The remaining part of this section is devoted to the proof of this theorem.

4.1. Setting up the circle method. We detect the summation condition $l_{1} m-l_{2} n-$ $q h=0$ by means of additive characters:

$$
\mathscr{D}\left(g, l_{1}, l_{2}, q\right)=\int_{\mathbb{R}} G(\alpha) 1_{[0,1]}(\alpha) d \alpha
$$

with

$G(\alpha):=H(\alpha) K(\alpha):=\sum_{h \neq 0} \phi(q h) e(-\alpha q h) \times \sum_{m, n \geq 1} \overline{\lambda_{g}}(m) \lambda_{g}(n) F\left(l_{1} m, l_{2} n\right) e\left(\alpha\left(l_{1} m-l_{2} n\right)\right)$.

As in [H03a, HM04a, B104b], we apply Jutila's method of overlapping intervals $[\mathrm{J} 92, \mathrm{~J} 96]$ to approximate the characteristic function of the unit interval $I(\alpha)=$ $1_{[0,1]}(\alpha)$ by sums of characteristic functions of intervals centered at well chosen rationals. Let $C \geq Y$ be a large parameter to be chosen later, and let $w$ be a smooth function supported on $[C / 2,3 C]$ with values in $[0,1]$ equal to 1 on $[C, 2 C]$ such that $w^{(i)}(x) \ll_{i} C^{-i}$. We also set

$$
\delta:=C^{-1}, \quad D^{\prime}:=D l_{1} l_{2}, \quad L:=\sum_{c \equiv 0\left(D^{\prime}\right)} w(c) \varphi(c), \quad P:=T C=q D \mu_{g} l_{1} l_{2} X Y Z C
$$

Note that, assuming $C \geq D^{\prime}, L$ satisfies the inequality

$$
L \gg_{\varepsilon} \frac{C^{2-\varepsilon}}{D l_{1} l_{2}}
$$

for any $\varepsilon>0$. The approximation to $I(\alpha)$ is provided by

$$
\tilde{I}(\alpha):=\frac{1}{2 \delta L} \sum_{c \equiv 0\left(D^{\prime}\right)} w(c) \sum_{\substack{a(\bmod c) \\(a, c)=1}} 1_{[a / c-\delta, a / c+\delta]}(\alpha)
$$

(which is supported in $[-1,2]$ ), and by the main theorem in [J92] one has 


$$
\int_{[-1,2]}|I(\alpha)-\tilde{I}(\alpha)|^{2} d \alpha \ll_{\varepsilon} \frac{C^{2+\varepsilon}}{\delta L^{2}} \ll_{\varepsilon} C^{3 \varepsilon} \frac{\left(D l_{1} l_{2}\right)^{2}}{C}
$$

Next, we introduce the corresponding approximation of $\mathscr{D}\left(g, l_{1}, l_{2}, q\right)$ :

$$
\tilde{\mathscr{D}}\left(g, l_{1}, l_{2}, q\right):=\int_{[-1,2]} G(\alpha) \tilde{I}(\alpha) d \alpha .
$$

Then it follows from (4.6) that

$$
\left|\mathscr{D}\left(g, l_{1}, l_{2}, q\right)-\tilde{\mathscr{D}}\left(g, l_{1}, l_{2}, q\right)\right| \leq\|I-\tilde{I}\|_{2}\|G\|_{2} \ll_{\varepsilon} C^{2 \varepsilon} \frac{D l_{1} l_{2}}{C^{1 / 2}}\|G\|_{2} .
$$

By Parseval,

$$
\|G\|_{2} \leq\|H\|_{2}\|K\|_{\infty} \ll\left(\frac{Y}{q}\right)^{1 / 2}\|K\|_{\infty},
$$

while an integration by parts shows that

$$
K(\alpha)=l_{1} l_{2} \int_{0}^{\infty} \int_{0}^{\infty} F^{(1,1)}\left(l_{1} x, l_{2} y\right) \overline{S_{g}\left(-l_{1} \alpha, x\right)} S_{g}\left(-l_{2} \alpha, y\right) d x d y
$$

so that by (4.1) and (4.2),

$$
\|K\|_{\infty} \ll_{\varepsilon} T^{2 \varepsilon} \omega_{g}^{2} D \mu_{g} Z^{2}\left(\frac{X Y}{l_{1} l_{2}}\right)^{1 / 2} .
$$

Collecting the above estimates, we find that

$$
\mathscr{D}-\tilde{\mathscr{D}} \ll_{\varepsilon} P^{2 \varepsilon} \omega_{g}^{2} D^{2} \mu_{g} Z^{2}\left(\frac{l_{1} l_{2} X Y^{2}}{q C}\right)^{1 / 2} .
$$

4.2. $\tilde{\mathscr{D}}$ as a sum of Kloosterman sums. We have

$$
\tilde{\mathscr{D}}=\frac{1}{L} \sum_{c \equiv 0\left(D^{\prime}\right)} w(c) \sum_{\substack{a(\bmod c) \\(a, c)=1}} \mathfrak{I}_{\delta, a / c}
$$

where

$$
\mathfrak{J}_{\delta, a / c}:=\sum_{h} e\left(\frac{-a q h}{c}\right) \sum_{m, n} \bar{\lambda}_{g}(m) \lambda_{g}(n) e\left(\frac{a l_{1} m}{c}\right) e\left(\frac{-a l_{2} n}{c}\right) E(m, n, h)
$$


and

$$
E(x, y, z):=F\left(l_{1} x, l_{2} y\right) \phi(q z) \frac{1}{2 \delta} \int_{-\delta}^{\delta} e\left(\alpha\left(l_{1} x-l_{2} y-q z\right)\right) d \alpha
$$

By applying Proposition 2.1 to the variables $m, n$ and by summing over $a, c$, we get (observe that the factor $\bar{\chi}_{g}(\bar{a})$ from the $m$-sum is cancelled by $\chi_{g}(\bar{a})$ coming from the $n$-sum) together with (2.5)

$$
\tilde{\mathscr{D}}=\sum_{ \pm, \pm} \varepsilon_{g}^{ \pm} \varepsilon_{g}^{ \pm} \tilde{\mathscr{D}}^{ \pm, \pm}
$$

where

$$
\tilde{\mathscr{D}}^{ \pm, \pm}:=\frac{1}{L} \sum_{m, n} \bar{\lambda}_{g}(m) \lambda_{g}(n) \sum_{c \equiv 0\left(D^{\prime}\right)} \sum_{h \neq 0} \frac{S\left(q h, \mp l_{1} m \pm l_{2} n ; c\right)}{c} \mathscr{E}^{ \pm}, \pm(m, n, h ; c),
$$

and

$$
\mathscr{E}^{ \pm, \pm}(m, n, h ; c):=\frac{l_{1} l_{2} w(c)}{c} \int_{0}^{\infty} \int_{0}^{\infty} E(x, y, h) J_{g}^{ \pm}\left(\frac{4 \pi l_{1} \sqrt{m x}}{c}\right) J_{g}^{ \pm}\left(\frac{4 \pi l_{2} \sqrt{n y}}{c}\right) d x d y
$$

and where $\varepsilon_{g}^{+}=1$ and $\varepsilon_{g}^{-}= \pm 1$ is the sign of $g$ if it is not induced from a holomorphic form, i.e., $\varepsilon_{g}^{-}$satisfies $\rho_{g}(-n)=\varepsilon_{g}^{-} \frac{\Gamma\left(\frac{1}{2}+i t+\frac{k}{2}\right)}{\Gamma\left(\frac{1}{2}+i t-\frac{k}{2}\right)} \rho_{g}(n)$ for all $n \geq 1$.

4.3. Estimates for $\mathscr{E}^{ \pm, \pm}$and its derivatives. Notice that the definition of $E$ and the various assumptions made so far imply that

$$
E(x, y, z)=0 \quad \text { unless } x \sim X / l_{1}, y \sim Y / l_{2},|q z| \leq 4 Y \text {. }
$$

Moreover,

$$
E^{(i, j, k)}(x, y, z) \ll_{i, j, k} \frac{Z^{i+j} l_{1}^{i} l_{2}^{j} q^{k}}{X^{i} Y^{j+k}}
$$

so that for any fixed $h$

$$
\left\|E^{(i, j, k)}(*, *, h)\right\|_{1} \ll_{i, j, k} \frac{Z^{i+j} l_{1}^{i-1} l_{2}^{j-1} q^{k} X Y}{X^{i} Y^{j+k}}
$$

and therefore

$$
\left\|E^{(i, j, k)}\right\|_{1} \ll_{i, j, k} \frac{Z^{i+j} l_{1}^{i-1} l_{2}^{j-1} q^{k-1} X Y^{2}}{X^{i} Y^{j+k}} .
$$


Next, we evaluate $\mathscr{E}^{ \pm, \pm}(m, n, h ; c)$ and its partial derivatives. Depending on the case, $\mathscr{E} \pm, \pm(m, n, h ; c)$ can be written as a linear combination (with constant coefficients) of integrals of the form

$$
\frac{l_{1} l_{2} w(c)}{c} \int_{0}^{\infty} \int_{0}^{\infty} E(x, y, h) J_{1, v_{1}}\left(\frac{4 \pi l_{1} \sqrt{m x}}{c}\right) J_{2, v_{2}}\left(\frac{4 \pi l_{2} \sqrt{n y}}{c}\right) d x d y
$$

where

$$
\left\{J_{1, v}(x), J_{2, v}(x)\right\} \subset\left\{\frac{Y_{v}(x)}{\operatorname{ch}(\pi t)}, \operatorname{ch}(\pi t) K_{v}(x)\right\}
$$

with $v \in\left\{ \pm 2 i t_{g}\right\}$ if $g$ is a Maass form of weight 0 and spectral parameter $t_{g}$; or

$$
\left\{J_{1, v}(x), J_{2, v}(x)\right\} \subset\left\{\frac{Y_{v}(x)}{\operatorname{sh}(\pi t)}, \operatorname{sh}(\pi t) K_{v}(x)\right\}
$$

with $v \in\left\{ \pm 2 i t_{g}\right\}$ if $g$ is a Maass form of weight 1 ; or

$$
J_{1, v}(x)=J_{2, v}(x)=J_{k_{g}-1}(x),
$$

if $g$ is a holomorphic form of weight $k_{g}$.

In order to estimate (4.12) efficiently, we integrate by parts $i$ (resp. $j$ ) times with respect to $x$ (resp. $y$ ), where $i$ (resp. $j$ ) will be determined later in terms of $m$ (resp. $n$ ) and $\varepsilon$. Using $(6.1)$, we see that $\mathscr{E} \pm, \pm(m, n, h ; c)$ can be written as a linear combination (with constant coefficients) of expressions of the form

$$
\begin{aligned}
\frac{l_{1} l_{2} w(c)}{c}\left(\frac{l_{1} \sqrt{m}}{c}\right)^{-2 i}\left(\frac{l_{2} \sqrt{n}}{c}\right)^{-2 j} \int_{0}^{\infty} & \int_{0}^{\infty} \frac{\partial^{i+j}}{\partial x^{i} \partial y^{j}}\left\{E(x, y, h) W_{1}^{-v_{1}} W_{2}^{-v_{2}}\right\} \\
& \times W_{1}^{v_{1}+i} W_{2}^{v_{2}+j} J_{1, v_{1}+i}\left(W_{1}\right) J_{2, v_{2}+j}\left(W_{2}\right) d x d y,
\end{aligned}
$$

where $\left\{v_{1}, v_{2}\right\} \subset\left\{ \pm 2 i t_{g}\right\}$ (or $\left.v_{1}, v_{2}=k_{g}-1\right)$ and

$$
W_{1}:=\frac{4 \pi l_{1} \sqrt{m x}}{c} \sim \frac{\sqrt{m l_{1} X}}{C}, \quad W_{2}:=\frac{4 \pi l_{2} \sqrt{n y}}{c} \sim \frac{\sqrt{n l_{2} Y}}{C}
$$

in view of (4.9). Using (4.11) and Proposition 6.2 in the slightly weaker form

$$
\begin{aligned}
& J_{1, v_{1}+i}\left(W_{1}\right) \ll_{i, \varepsilon} \mu_{g}^{i+\varepsilon}\left(1+W_{1}^{-1}\right)^{i+2\left|\Im t_{g}\right|+\varepsilon}\left(1+W_{1}\right)^{-1 / 2}, \\
& J_{2, v_{2}+j}\left(W_{2}\right) \ll_{j, \varepsilon} \mu_{g}^{j+\varepsilon}\left(1+W_{2}^{-1}\right)^{j+2\left|\Im t_{g}\right|+\varepsilon}\left(1+W_{2}\right)^{-1 / 2},
\end{aligned}
$$


we can deduce for any $i, j \geq 0$ that

$$
\begin{aligned}
\mathscr{E}^{ \pm, \pm}(m, n, h ; c) \ll_{i, j, \varepsilon} & P^{\varepsilon}\left(\mu_{g}^{2} Z\right)^{i+j}\left\{\frac{C^{2}}{l_{1} m X}+\left(\frac{C^{2}}{l_{1} m X}\right)^{1 / 2}\right\}^{i} \\
& \times\left\{\frac{C^{2}}{l_{2} n Y}+\left(\frac{C^{2}}{l_{2} n Y}\right)^{1 / 2}\right\}^{j} \Xi(m, n),
\end{aligned}
$$

where

$$
\Xi(m, n):=\frac{X Y}{C}\left\{\left(1+\frac{C^{2}}{l_{1} m X}\right)\left(1+\frac{C^{2}}{l_{2} n Y}\right)\right\}^{\left|\Im t_{g}\right|}\left\{\left(1+\frac{l_{1} m X}{C^{2}}\right)\left(1+\frac{l_{2} n Y}{C^{2}}\right)\right\}^{-1 / 4} .
$$

This shows, upon choosing $i$ and $j$ appropriately, that $\mathscr{E}^{ \pm, \pm}(m, n, h ; c)$ is very small unless

$$
q|h| \leq 4 Y, \quad c \sim C, \quad m \ll_{\varepsilon} P^{\varepsilon} \frac{\mu_{g}^{4} Z^{2} C^{2}}{l_{1} X}, \quad n \ll_{\varepsilon} P^{\varepsilon} \frac{\mu_{g}^{4} Z^{2} C^{2}}{l_{2} Y},
$$

and in this range we retain the bound (by taking $i=j=0$ )

$$
\mathscr{E}^{ \pm, \pm}(m, n, h ; c) \ll_{\varepsilon} P^{\varepsilon} \Xi(m, n) .
$$

The partial derivatives

$$
m^{i} n^{j} h^{k} c^{l} \frac{\partial^{i+j+k+l}}{\partial m^{i} \partial n^{j} \partial h^{k} \partial c^{l}} \mathscr{E}^{ \pm, \pm}(m, n, h ; c)
$$

can be estimated similarly. We shall restrict our attention to the range (4.14). The same argument as above yields that outside this range the partial derivatives are very small. By (6.1) applied to the $m$ and $n$ variables and (6.2) applied to the $c$ variable, the above partial derivative is a linear combination of integrals of the form

$R_{l}\left(t_{g}\right) c^{a_{3}} \frac{\partial^{a_{3}}}{\partial c^{a_{3}}}\left(\frac{w(c)}{c}\right) \int_{0}^{\infty} \int_{0}^{\infty} h^{k} \frac{\partial^{k}}{\partial h^{k}} E(x, y, h) W_{1}^{a_{1}} W_{2}^{a_{2}} J_{1, v_{1}-a_{1}}\left(W_{1}\right) J_{2, v_{2}-a_{2}}\left(W_{2}\right) d x d y$

where $R_{l}$ is a polynomial of degree $\leq l$ and the nonnegative integers $a_{1}, a_{2}, a_{3}$ satisfy

$$
a_{1}+a_{2}+a_{3} \leq i+j+l \text {. }
$$

Therefore we obtain using (4.14) 


$$
\begin{aligned}
& m^{i} n^{j} h^{k} c^{l}(\mathscr{E} \pm, \pm)^{(i, j, k, l)}(m, n, h ; c) \\
& \ll_{i, j, k, l, \varepsilon} P^{\varepsilon}\left(\frac{q|h|}{Y}\right)^{k} \mu_{g}^{l}\left(1+\frac{\sqrt{l_{1} m X}}{C}+\frac{\sqrt{l_{2} n Y}}{C}\right)^{i+j+l} \Xi(m, n) \\
& \ll_{i, j, k, l, \varepsilon} P^{\varepsilon} \mu_{g}^{l}\left(P^{\varepsilon} \mu_{g}^{2} Z\right)^{i+j+l} \Xi(m, n)
\end{aligned}
$$

4.4. Bounding $\tilde{\mathscr{D}}^{ \pm} \pm$via the large sieve. We only treat $\tilde{\mathscr{D}}^{-,-}$, the other terms being similar. To simplify notation, we rename $\tilde{\mathscr{D}}^{-,-}$as $\tilde{\mathscr{D}}$ and $\mathscr{E}^{-,-}$as $\mathscr{E}$. We collect the terms in the definition (4.8) according to

$$
h^{\prime}:=l_{1} m-l_{2} n
$$

Thus we have the natural splitting

$$
\tilde{\mathscr{D}}=\tilde{\mathscr{D}}^{0}+\tilde{\mathscr{D}}^{+}+\tilde{\mathscr{D}}^{-},
$$

where

$$
\tilde{\mathscr{D}}^{0}:=\frac{1}{L_{l_{1}}} \sum_{m=l_{2} n} \bar{\lambda}_{g}(m) \lambda_{g}(n) \sum_{c \equiv 0\left(D^{\prime}\right)} \sum_{h \neq 0} \frac{r(q h ; c)}{c} \mathscr{E}(m, n, h ; c)
$$

with

$$
r(q h ; c)=S(q h, 0 ; c)=\sum_{c^{\prime} \mid(q h, c)} \mu\left(c / c^{\prime}\right) c^{\prime}
$$

the Ramanujan sum, and

$$
\tilde{\mathscr{D}}^{ \pm}:=\frac{1}{L} \sum_{c \equiv 0} \sum_{\left(D^{\prime}\right)} \sum_{h \neq 0} \frac{S\left(q h, h^{\prime} ; c\right)}{c} \sum_{l_{1} m-l_{2} n=h^{\prime}} \bar{\lambda}_{g}(m) \lambda_{g}(n) \mathscr{E}(m, n, h ; c)
$$

4.4.1. Bounding $\tilde{\mathscr{D}}^{0}$. We set $l_{1}^{\prime}:=l_{1} /\left(l_{1}, l_{2}\right), l_{2}^{\prime}:=l_{2} /\left(l_{1}, l_{2}\right)$; then

$$
\tilde{\mathscr{D}}^{0}=\frac{1}{L} \sum_{m \geq 1} \overline{\lambda_{g}}\left(l_{2}^{\prime} m\right) \lambda_{g}\left(l_{1}^{\prime} m\right) \sum_{c \equiv 0\left(D^{\prime}\right)} \frac{1}{c} \sum_{h \neq 0} r(q h ; c) \mathscr{E}\left(l_{2}^{\prime} m, l_{1}^{\prime} m, h ; c\right) .
$$

The $c$-sum equals

$$
\sum_{c^{\prime \prime}} \frac{\mu\left(c^{\prime \prime}\right)}{c^{\prime \prime}} \sum_{\left(D^{\prime} /\left(c^{\prime \prime}, D^{\prime}\right)\right) \mid c^{\prime}} \sum_{h \neq 0} \mathscr{E}\left(l_{2}^{\prime} m, l_{1}^{\prime} m, \frac{c^{\prime}}{\left(c^{\prime}, q\right)} h ; c^{\prime} c^{\prime \prime}\right),
$$


therefore by (4.14) and (4.15) it is bounded by

$$
\ll_{\varepsilon} P^{\varepsilon} \frac{Y}{q} \Xi\left(l_{2}^{\prime} m, l_{1}^{\prime} m\right) \sum_{c^{\prime \prime}} \sum_{\begin{array}{c}
\left(D^{\prime} /\left(c^{\prime \prime}, D^{\prime}\right)\right) \mid c^{\prime} \\
c^{\prime} c^{\prime \prime} \sim C
\end{array}} \frac{\mu\left(c^{\prime \prime}\right)\left(c^{\prime}, q\right)}{c^{\prime} c^{\prime \prime}} \ll P^{\varepsilon} \frac{\left(q, D l_{1} l_{2}\right)}{D l_{1} l_{2}} \frac{Y}{q} \Xi\left(l_{2}^{\prime} m, l_{1}^{\prime} m\right) .
$$

In summing over the $m$ variable we may restrict ourselves to the range

$$
\left[l_{1}, l_{2}\right] m \ll_{\varepsilon} P^{\varepsilon} \mu_{g}^{4} Z^{2}\left(C^{2} / Y\right)
$$

as the remaining contribution is negligible. If $Y / X \ll_{\varepsilon} P^{\varepsilon} \mu_{g}^{4} Z^{2}$, then we split the $m$-sum into three parts,

$$
\sum_{\left[l_{1}, l_{2}\right] m<C^{2} / Y} \cdots+\sum_{C^{2} / Y \leq\left[l_{1}, l_{2}\right] m<C^{2} / X} \cdots+\sum_{C^{2} / X \leq\left[l_{1}, l_{2}\right] m \ll{ }_{2} P^{\varepsilon} \mu_{g}^{4} Z^{2}\left(C^{2} / Y\right)} \cdots
$$

and combine (4.5) and (4.13) with basic properties of the Hecke eigenvalues $\lambda_{g}(n)$ to infer that

$$
\tilde{\mathscr{D}}^{0} \ll_{\varepsilon} P^{2 \varepsilon} \frac{\left(q, D l_{1} l_{2}\right)}{q\left[l_{1}, l_{2}\right]^{1-\theta}} \frac{X Y^{2}}{C}\left(X^{-\theta} Y^{\theta-1}+X^{-3 / 4} Y^{-1 / 4}+\mu_{g}^{2} Z X^{-1 / 4} Y^{-3 / 4}\right) .
$$

If $Y / X \gg{ }_{\varepsilon} P^{\varepsilon} \mu_{g}^{4} Z^{2}$, then we split the $m$-sum into two parts,

$$
\sum_{\left[l_{1}, l_{2}\right] m<C^{2} / Y} \cdots+\sum_{C^{2} / Y \leq\left[l_{1}, l_{2}\right] m \ll{ }_{\varepsilon} P^{\varepsilon} \mu_{g}^{4} Z^{2}\left(C^{2} / Y\right)} \cdots
$$

and infer similarly that

$$
\tilde{\mathscr{D}}^{0} \ll_{\varepsilon} P^{2 \varepsilon} \frac{\left(q, D l_{1} l_{2}\right)}{q\left[l_{1}, l_{2}\right]^{1-\theta}} \frac{X Y^{2}}{C}\left(X^{-\theta} Y^{\theta-1}+\mu_{g}^{3-4 \theta} Z^{3 / 2-2 \theta} X^{-\theta} Y^{\theta-1}\right)
$$

In both cases we conclude that

$$
\tilde{\mathscr{D}}^{0} \ll_{\varepsilon} P^{3 \varepsilon} \mu_{g}^{2} Z \frac{X^{3 / 4} Y^{5 / 4}}{C}
$$

4.4.2. Bounding $\tilde{\mathscr{D}}^{ \pm}$. Following [B104b], we decompose the inner sum in (4.17) as

$$
\int_{1}^{\infty} \sum_{\substack{n \leq y \\ l_{1} m-l_{2} n=h^{\prime}}} \overline{\lambda_{g}}(m) \lambda_{g}(n) \frac{\partial}{\partial y} \mathscr{E}(x, y, h ; c) d y
$$


where the variables $x$ and $y$ are connected by the equation

(4.20) $l_{1} x-l_{2} y=h^{\prime}$

(note that $h^{\prime}, y>0$ implies $x>0$ ). Then $\tilde{\mathscr{D}}^{+}$decomposes accordingly as

$$
\tilde{\mathscr{D}}^{+}=\frac{1}{L} \int_{1}^{\infty} \tilde{\mathscr{D}}^{+}(y) d y
$$

where

$$
\tilde{\mathscr{D}}^{+}(y):=\sum_{c \equiv 0\left(D^{\prime}\right)} \sum_{h \neq 0} \sum_{h^{\prime}>0} b_{y, h^{\prime}} \frac{S\left(q h, h^{\prime} ; c\right)}{c} g_{y}\left(q h, h^{\prime} ; c\right)
$$

and

$$
b_{y, h^{\prime}}:=\sum_{\substack{n \leq y \\ l_{1} m-l_{2} n=h^{\prime}}} \bar{\lambda}_{g}(m) \lambda_{g}(n), \quad g_{y}\left(h, h^{\prime} ; c\right):=\frac{\partial}{\partial y} \mathscr{E}\left(\frac{l_{2} y+h^{\prime}}{l_{1}}, y, \frac{h}{q} ; c\right) .
$$

In particular, $g_{y}\left(h, h^{\prime} ; c\right)$ and all its partial derivatives are very small unless

$$
|h| \leq 4 Y, \quad c \sim C, \quad x \ll_{\varepsilon} P^{\varepsilon} \frac{\mu_{g}^{4} Z^{2} C^{2}}{l_{1} X}, \quad y \ll_{\varepsilon} P^{\varepsilon} \frac{\mu_{g}^{4} Z^{2} C^{2}}{l_{2} Y}
$$

and in this range they obey by (4.16) and (4.20) the bound

$$
\begin{aligned}
& h^{i} h^{\prime} c^{k} g_{y}^{(i, j, k)}\left(h, h^{\prime} ; c\right) \\
&=\left(\frac{h}{q}\right)^{i}\left(\frac{h^{\prime}}{l_{1}}\right)^{j} c^{k}\left\{\frac{l_{2}}{l_{1}} \mathscr{E}^{(1+j, 0, i, k)}\left(x, y, \frac{h}{q} ; c\right)+\mathscr{E}^{(j, 1, i, k)}\left(x, y, \frac{h}{q} ; c\right)\right\} \\
& \ll i, j, k, \varepsilon \\
& \mu_{g}^{2+2 j+3 k}\left(P^{\varepsilon} Z\right)^{1+j+k} y^{-1} \Xi(x, y) .
\end{aligned}
$$

Using the definition (4.13) it can be checked that in the range (4.24) we have the uniform bound

$$
(x y)^{1 / 2} \Xi(x, y) \ll_{\varepsilon} P^{\varepsilon} \mu_{g}^{2} Z C\left(\frac{X Y}{l_{1} l_{2}}\right)^{1 / 2}=: W
$$

so that in fact 


$$
h^{i} h^{\prime j} c^{k} g_{y}^{(i, j, k)}\left(h, h^{\prime} ; c\right) \ll_{i, j, k, \varepsilon} \mu_{g}^{2+2 j+3 k}\left(P^{\varepsilon} Z\right)^{1+j+k} W x^{-1 / 2} y^{-3 / 2} .
$$

This also shows that we can decompose $g_{y}\left(h, h^{\prime} ; c\right)$ dyadically in the $h$ and $h^{\prime}$ variables such that

$$
g_{y}\left(h, h^{\prime} ; c\right)=\sum_{H, H^{\prime} \geq 1} g_{y, H, H^{\prime}}\left(h, h^{\prime} ; c\right),
$$

where $H$ and $H^{\prime}$ run through the powers of 2 independently, and $g_{y, H, H^{\prime}}\left(h, h^{\prime} ; c\right)$ as a function of $h$ (resp. $h^{\prime}$ ) is supported on $H / 2 \leq|h| \leq 2 H$ (resp. $\left.H^{\prime} / 2 \leq h^{\prime} \leq 2 H^{\prime}\right)$ and satisfies

$$
g_{y, H, H^{\prime}}^{(i, j, k)}\left(h, h^{\prime} ; c\right) \ll_{i, j, k, \varepsilon} \frac{P^{\varepsilon} \mu_{g}^{2} Z W}{\tilde{x}^{1 / 2} y^{3 / 2}} \cdot \frac{\tilde{Z}^{j+k}}{H^{i} H^{\prime j} C^{k}},
$$

where

$$
\tilde{Z}:=P^{\varepsilon} \mu_{g}^{3} Z
$$

and $\tilde{x}$ is connected to $y$ by the equation

$$
l_{1} \tilde{x}-l_{2} y=H^{\prime}
$$

The same argument also shows that all these partial derivatives are very small unless

$$
H \leq 8 Y, \quad H^{\prime} \ll_{\varepsilon} P^{\varepsilon} \frac{\mu_{g}^{4} Z^{2} C^{2}}{X}, \quad c \sim C, \quad y \ll_{\varepsilon} P^{\varepsilon} \frac{\mu_{g}^{4} Z^{2} C^{2}}{l_{2} Y} .
$$

Accordingly, $\tilde{\mathscr{D}}^{+}(y)$ of (4.22) decomposes into a double sum over the $H$ and the $H^{\prime}$. We further decompose all the pieces according to the $q$-part of the $h$ variable and we find (after replacing $q h$ by $h$ ) that

$$
\tilde{\mathscr{D}}^{+}(y)=\sum_{H, H^{\prime} \geq 1} \sum_{q^{\prime} \mid q^{\infty}} \tilde{\mathscr{D}}_{H, H^{\prime}, q^{\prime}}^{+}(y)
$$

where

$$
\tilde{\mathscr{D}}_{H, H^{\prime}, q^{\prime}}^{+}(y):=\sum_{c \equiv 0\left(D^{\prime}\right)} \sum_{\begin{array}{c}
q q^{\prime} \mid h \\
\left(h / q q^{\prime}, q q^{\prime}\right)=1
\end{array}} \sum_{h^{\prime}>0} b_{y, h^{\prime}} \frac{S\left(h, h^{\prime} ; c\right)}{c} g_{y, H, H^{\prime}}\left(h, h^{\prime} ; c\right) .
$$

We are ready to apply the large sieve for the sums $D_{H, H^{\prime}, q^{\prime}}^{+}(y)$. By Theorem 4 and (4.26), 


$$
\begin{aligned}
\tilde{\mathscr{D}}_{H, H^{\prime}, q^{\prime}}^{+}(y) \ll_{\varepsilon} \frac{P^{\varepsilon} \mu_{g}^{2} Z W}{\tilde{x}^{1 / 2} y^{3 / 2}}\left(q^{\prime} P\right)^{2 \varepsilon}\left(q q^{\prime}\right)^{\theta}\left(1+\frac{C}{\sqrt{H H^{\prime}}}\right)^{2 \theta} \\
\times \tilde{Z}^{2}\left(\tilde{Z}^{3 / 2}+\tilde{Z} \frac{\sqrt{H H^{\prime}}}{C}+\tilde{Z}^{2 \theta} \frac{\left(q q^{\prime}, D^{\prime}\right) H}{q q^{\prime} D^{\prime}}\right)^{1 / 2} \\
\times\left(\tilde{Z}^{3 / 2}+\tilde{Z} \frac{\sqrt{H H^{\prime}}}{C}+\tilde{Z}^{2 \theta} \frac{H^{\prime}}{D^{\prime}}\right)^{1 / 2}\left(\frac{H}{q q^{\prime}}\right)^{1 / 2}\left\|b_{y}\right\|_{2} .
\end{aligned}
$$

Here, by (4.23) and (4.4),

$$
\left\|b_{y}\right\|_{2} \ll_{\varepsilon} P^{\varepsilon} \omega_{g} D^{1 / 2} \mu_{g}^{1 / 2}(\tilde{x} y)^{1 / 2} .
$$

If we choose $C \geq \tilde{Z}^{2} D^{\prime} Y$, then by our general assumption $Y \geq X$ it follows that in the range (4.28)

$$
\left(1+\frac{C}{\sqrt{H H^{\prime}}}\right)^{2 \theta}\left(\tilde{Z}^{3 / 2}+\tilde{Z} \frac{\sqrt{H H^{\prime}}}{C}+\tilde{Z}^{2 \theta} \frac{H^{\prime}}{D^{\prime}}\right)^{1 / 2}\left(\frac{H}{q q^{\prime}}\right)^{1 / 2} \ll_{\varepsilon} P^{\varepsilon} \mu_{g}^{2} Z \tilde{Z}^{\theta} C\left(\frac{Y}{q q^{\prime} D^{\prime} X}\right)^{1 / 2} .
$$

These additional estimates yield (for the relevant range (4.28))

$$
\begin{aligned}
\tilde{\mathscr{D}}_{H, H^{\prime}, q^{\prime}}^{+}(y) \ll_{\varepsilon} y^{-1}\left(q^{\prime} P\right)^{5 \varepsilon} \omega_{g} D^{1 / 2} \mu_{g}^{11 / 2} Z^{5 / 2} \tilde{Z}^{11 / 4+\theta} C W\left(q q^{\prime}\right)^{\theta} \\
\times\left(\frac{Y}{q q^{\prime} D^{\prime} X}\right)^{1 / 2}\left(\left(\frac{Y}{X}\right)^{1 / 2}+\frac{\left(q q^{\prime}, D^{\prime}\right)}{q q^{\prime} D^{\prime}} Y\right)^{1 / 2},
\end{aligned}
$$

and by the definition of $W$ and $\tilde{Z}$ (see (4.25) and (4.27)) the right hand side is

$$
\ll_{\varepsilon} y^{-1} \frac{C^{2}}{l_{1} l_{2}}\left(q^{\prime} P\right)^{11 \varepsilon} \omega_{g} \mu_{g}^{63 / 4+3 \theta} Z^{25 / 4+\theta}\left(q q^{\prime}\right)^{\theta-1 / 2} Y\left(\left(\frac{Y}{X}\right)^{1 / 2}+\frac{\left(q, D^{\prime}\right)}{q D^{\prime}} Y\right)^{1 / 2} .
$$

Finally, by (4.29), (4.28), (4.21) and (4.5) we conclude that

$$
\tilde{\mathscr{D}}^{+} \ll_{\varepsilon} P^{12 \varepsilon} \omega_{g} D \mu_{g}^{63 / 4+3 \theta} Z^{25 / 4+\theta} q^{\theta-1 / 2} Y\left(\left(\frac{Y}{X}\right)^{1 / 2}+\frac{\left(q, D l_{1} l_{2}\right)}{q D l_{1} l_{2}} Y\right)^{1 / 2} .
$$

The treatment of $\tilde{\mathscr{D}}^{-}$is very similar to that of $\tilde{\mathscr{D}}^{+}$except that instead of (4.19) we decompose the inner sum in (4.17) as

$$
\int_{1}^{\infty} \sum_{\substack{m \leq x \\ l_{1} m-l_{2} n=h^{\prime}}} \overline{\lambda_{g}}(m) \lambda_{g}(n) \frac{\partial}{\partial x} \mathscr{E}(x, y, h ; c) d x
$$


4.5. Concluding Theorem 5. Theorem 5 follows immediately from (4.7), (4.18) and (4.30) upon choosing $C$ to be a large power of $T$, say

$$
C:=\left(q D \mu_{g} l_{1} l_{2} X Y Z\right)^{100} .
$$

\section{Application to subconvexity bounds for twisted $L$-functions}

Let $g$ be a cuspidal newform (i.e., either a holomorphic or a Maass cusp form) as in (1.1), let $\Re s=\frac{1}{2}$, and let $\chi$ be a primitive character of conductor $q$.

5.1. Approximate functional equation. Using the functional equation of the $L$-function attached to the cuspidal automorphic representation $\pi_{g} \otimes \chi=$ $\bigotimes_{v}\left(\pi_{g, v} \otimes \chi_{v}\right)$ and a standard technique involving Mellin transforms, we can express the special value $L(g \otimes \chi, s)=L\left(\pi_{g} \otimes \chi, s\right)$ as a sum of two Dirichlet series of essentially $\sqrt{C}$ terms, where

$$
C:=C\left(\pi_{g} \otimes \chi, s\right) \leq|s|^{2} \mu_{g}^{2} D q^{2}
$$

is the analytic conductor defined by [IS00]. For example, Theorem 1 in [H02] shows that

$$
L(g \otimes \chi, s)=\sum_{n \geq 1} \frac{\lambda_{\pi_{g} \otimes \chi}(n)}{n^{s}} W\left(\frac{n}{C^{1 / 2}}\right)+\kappa \sum_{n \geq 1} \frac{\overline{\lambda_{\pi_{g} \otimes \chi}}(n)}{n^{1-s}} \bar{W}\left(\frac{n}{C^{1 / 2}}\right),
$$

where $\kappa$ is of modulus one, $\lambda_{\pi_{g} \otimes \chi}(n)$ are the coefficients of the Dirichlet series $L(g \otimes \chi, s)$, and $W$ is a smooth bounded function of $(0, \infty)$ (depending on $\pi_{g} \otimes \chi$ and $s$ ) satisfying the uniform estimates

$$
x^{i} W^{(i)}(x) \ll_{i, A}(1+x)^{-A}
$$

for any $x>0$, any integer $i \geq 0$ and any $A \geq 0$ with implied constants depending only on $i$ and $A$. The coefficient $\lambda_{\pi_{g} \otimes \chi}(n)$ may be complicated for $n$ not coprime with $q D$, however for $\Re s>1$ one has

$$
L(g \otimes \chi, s)=\prod_{p \mid q D} \frac{1-\lambda_{g}(p) \chi(p) p^{-s}+\chi\left(p^{2}\right) p^{-2 s}}{L^{-1}\left(\pi_{g, p} \otimes \chi_{p}, s\right)} \sum_{n \geq 1} \frac{\lambda_{g}(n) \chi(n)}{n^{s}}
$$

so that by $(2.4)$

$$
\sum_{n \geq 1} \frac{\lambda_{\pi_{g} \otimes \chi}(n)}{n^{s}} W\left(\frac{n}{C^{1 / 2}}\right) \ll_{\varepsilon} \sum_{d \mid(q D)^{\infty}} \frac{1}{d^{1 / 2-\theta-\varepsilon}}\left|\sum_{n \geq 1} \frac{\lambda_{g}(n) \chi(n)}{n^{s}} W\left(\frac{d n}{C^{1 / 2}}\right)\right|
$$

for any $\varepsilon>0$, the implied constant depending only on $\varepsilon$. 
By the rapid decay properties of $W$, the contribution of the $d, n$ such that $d n \geq C^{1 / 2+\varepsilon}$ is negligible for any given $\varepsilon>0$. For the rest of the sum we apply a smooth dyadic decomposition, so that we are left with estimating $O(\log C)$ sums of the form

$$
N^{-1 / 2} \Sigma(g \otimes \chi, N)=N^{-1 / 2} \sum_{n \geq 1} \lambda_{g}(n) \chi(n) W_{N, s}(n),
$$

where $1 \leq N \leq C^{1 / 2+\varepsilon}$ and $W_{N, s}$ is some smooth function supported in $[N, 2 N]$ satisfying

$$
x^{i} W_{N, s}^{(i)}(x) \ll_{i}|s|^{i}
$$

the implied constant depending only on $i$.

5.2. Amplification. We shall estimate the sums $\Sigma(g \otimes \chi, N)$ by the amplification method of [DFI93]. That is, we choose some $L$ so that $\log L \asymp \log q$ and estimate the amplified second moment

$$
S(g, N):=\sum_{\chi^{\prime}(\bmod q)}\left|\sum_{L \leq l \leq 2 L} \bar{\chi}(l) \chi^{\prime}(l)\right|^{2}\left|\Sigma\left(g \otimes \chi^{\prime}, N\right)\right|^{2},
$$

where $\chi^{\prime}$ runs over the characters modulo $q$ and

$$
\Sigma\left(g \otimes \chi^{\prime}, N\right):=\sum_{n \geq 1} \lambda_{g}(n) \chi^{\prime}(n) W_{N, s}(n) .
$$

By orthogonality of characters one has (see [M04b])

$$
S(g, N) \leq \varphi(q) \sum_{L \leq l_{1}, l_{2} \leq 2 L} \bar{\chi}\left(l_{1}\right) \chi\left(l_{2}\right) \sum_{h} \sum_{l_{1} m-l_{2} n=h q} \overline{\lambda_{g}}(m) \lambda_{g}(n) F\left(l_{1} m, l_{2} n\right),
$$

where

$$
F(x, y):=\overline{W_{N, s}\left(x / l_{1}\right)} W_{N, s}\left(y / l_{2}\right) .
$$

The total contribution of $h=0$ can be estimated by Cauchy-Schwarz and the Rankin-Selberg bound:

$$
\sum_{\substack{L \leq l_{1}, l_{2} \leq 2 L \\ l_{1} m=l_{2} n}} \overline{\lambda_{g}}(m) \lambda_{g}(n) F\left(l_{1} m, l_{2} n\right) \ll_{\varepsilon} T^{\varepsilon} N L, \quad T:=|s| \mu_{g} D q .
$$

For each pair $l_{1}, l_{2}$ coprime with $q$, the contribution of $h \neq 0$ can be estimated directly by Theorem 5 with the parameters (when $\left.l_{1} \leq l_{2}\right) X=l_{1} N, Y=l_{2} N, Z=|s|$ : 


$$
\sum_{h \neq 0} \sum_{l_{1} m-l_{2} n=h q} \bar{\lambda}_{g}(m) \lambda_{g}(n) F\left(l_{1} m, l_{2} n\right) \ll_{\varepsilon} T^{\varepsilon} U q^{\theta-1 / 2} N L\left(1+\frac{N}{q L}\right)^{1 / 2}
$$

where we have put for convenience

$$
U:=|s|^{25 / 4+\theta} \omega_{g} \mu_{g}^{63 / 4+3 \theta} D
$$

We also note that

$$
N \leq T^{\varepsilon} V q, \quad V:=|s| \mu_{g} D^{1 / 2}
$$

whence the obvious lower bound

$$
N^{-1 / 2} \Sigma(g \otimes \chi, N) \ll_{\varepsilon} q^{\varepsilon}\left(\frac{S(g, N)}{N L^{2}}\right)^{1 / 2}
$$

together with the above estimates imply that

$$
L(g \otimes \chi, s) \ll_{\varepsilon} T^{4 \varepsilon}\left\{\frac{q}{L}+U q^{1 / 2+\theta} L\left(1+\frac{V}{L}\right)^{1 / 2}\right\}^{1 / 2} .
$$

The expression on the right hand side suggests that we choose $L$ of the form

$$
L:=q^{(1-2 \theta) / 4} / R
$$

where $R \gg 1$ depends only on $s, \mu_{g}$ and $D$. If $R$ is not too large then we can guarantee with the help of the convexity bound

$$
L(g \otimes \chi, s) \ll_{\varepsilon} T^{\varepsilon} V^{1 / 2} q^{1 / 2}
$$

that $L \geq q^{\delta}$ with some $\delta>0$. More precisely, the bound (5.3) follows from the convexity bound unless

$$
U q^{1 / 2+\theta} L\left(1+\frac{V}{L}\right)^{1 / 2} \ll V q
$$

which by (5.4) is equivalent to

$$
\max \left\{\frac{U}{V R^{2}},\left(\frac{U^{2}}{V R^{4}}\right)^{1 / 3}\right\} \ll L .
$$

Fixing any $\delta>0$, this inequality can be rewritten as 


$$
\max \left\{\frac{U}{V R^{2}},\left(\frac{U^{2}}{V R^{4}}\right)^{1 / 3}\right\}^{1-\delta}\left(\frac{q^{(1-2 \theta) / 4}}{R}\right)^{\delta} \ll L,
$$

which implies that

$$
R^{\delta /(1-\delta)} \ll \max \left\{\frac{U}{V R^{2}},\left(\frac{U^{2}}{V R^{4}}\right)^{1 / 3}\right\} \Rightarrow q^{\delta(1-2 \theta) / 4} \ll L .
$$

This justifies the choice

$$
R:=\left(\frac{U^{2}}{V}\right)^{(1-\delta) /(4-\delta)}
$$

since then (5.3) holds true either because $L$ is admissible or as a consequence of the convexity bound.

5.3. Concluding Theorem 1. We choose $L$ according to (5.4)-(5.5), where $\delta$ is a very small positive number depending on $\varepsilon$ and $U, V$ are defined in (5.1)-(5.2). Then (5.3) implies that

$$
L(g \otimes \chi, s) \ll_{\varepsilon} T^{4 \varepsilon}\left\{U V^{1 / 2} q^{1 / 2+\theta} L\right\}^{1 / 2} \ll_{\varepsilon} T^{5 \varepsilon} U^{1 / 4} V^{3 / 8} q^{(3+2 \theta) / 8} .
$$

In view of (4.3), this is the bound of Theorem 1 .

\section{Appendix 1: Bounds for Bessel functions}

In this appendix we recall some facts about Bessel functions. Proofs of Propositions 6.1 and 6.2 can be found in the Appendix of [HM04a].

For $s \in \mathbb{C}$, the Bessel functions satisfy the recurrence relations

$$
\left(x^{s} J_{s}(x)\right)^{\prime}=x^{s} J_{s-1}(x), \quad\left(x^{s} Y_{s}(x)\right)^{\prime}=x^{s} Y_{s-1}(x), \quad\left(x^{s} K_{s}(x)\right)^{\prime}=-x^{s} K_{s-1}(x) .
$$

In particular, if $r>0$ and $H_{s}$ denotes either $J_{s}, Y_{s}$ or $K_{s}$, then

$$
\frac{d}{d x}\left((r \sqrt{x})^{s+1} H_{s+1}(r \sqrt{x})\right)= \pm\left(r^{2} / 2\right)(r \sqrt{x})^{s} H_{s}(r \sqrt{x})
$$

and for any $j \geq 0$,

$$
x^{j} \frac{d^{j}}{d^{j} x} H_{s}\left(\frac{r}{x}\right)=Q_{j}(s) H_{s}\left(\frac{r}{x}\right)+Q_{j-1}(s)\left(\frac{r}{x}\right)^{1} H_{s-1}\left(\frac{r}{x}\right)+\cdots+Q_{0}(s)\left(\frac{r}{x}\right)^{j} H_{s-j}\left(\frac{r}{x}\right),
$$

where each $Q_{i}$ is a polynomial of degree $i$ whose coefficients depend only on $i$ and $j$. 
Proposition 6.1. For any integer $k \geq 1$, the following uniform estimate holds:

$$
J_{k-1}(x) \ll \begin{cases}\frac{x^{k-1}}{2^{k-1} \Gamma\left(k-\frac{1}{2}\right)}, & 0<x \leq 1 \\ k x^{-1 / 2}, & 1<x .\end{cases}
$$

The implied constant is absolute.

Proposition 6.2. For any $\sigma>0$ and any $\varepsilon>0$, the following uniform estimates hold in the strip $|\Re s| \leq \sigma$ :

$$
\begin{aligned}
e^{-\pi|\Im s| / 2} Y_{s}(x) \ll \begin{cases}(1+|\Im s|)^{\sigma+\varepsilon} x^{-\sigma-\varepsilon}, & 0<x \leq 1+|\Im s| ; \\
(1+|\Im s|)^{-\varepsilon} x^{\varepsilon}, & 1+|\Im s|<x \leq 1+|s|^{2} ; \\
x^{-1 / 2}, & 1+|s|^{2}<x .\end{cases} \\
e^{\pi|\Im s| / 2} K_{s}(x) \ll \begin{cases}(1+|\Im s|)^{\sigma+\varepsilon} x^{-\sigma-\varepsilon}, & 0<x \leq 1+\pi|\Im s| / 2 ; \\
e^{-x+\pi|\Im s| / 2} x^{-1 / 2}, & 1+\pi|\Im s| / 2<x .\end{cases}
\end{aligned}
$$

The implied constants depend only on $\sigma$ and $\varepsilon$.

\section{Appendix 2: Improved bound for the Fourier coefficients of holomorphic half-integral weight cusp forms}

\section{By Zhengyu Mao}

In this appendix we apply the estimate obtained in Theorem 1 along with the work of [BM05] to get an improved upper bound for the Fourier coefficients of holomorphic half-integral weight cusp forms. For positive integers $k$ and $M$ and an even Dirichlet character $\chi$ modulo $4 M$ let $S_{k+1 / 2}(4 M, \chi)$ denote the space of holomorphic forms of weight $k+\frac{1}{2}$, level $M$, and nebentypus $\chi$. The functions $f(z)$ in this space satisfy (cf. [Wa81])

$$
f\left(\frac{a z+b}{c z+d}\right)=j(\sigma, z)^{2 k+1} \chi(d) f(z), \quad \sigma=\left(\begin{array}{ll}
a & b \\
c & d
\end{array}\right) \in \Gamma_{0}(4 M)
$$

where

$$
j(\sigma, z):=\theta\left(\frac{a z+b}{c z+d}\right) / \theta(z), \quad \theta(z):=\sum_{n=-\infty}^{\infty} e\left(n^{2} z\right) .
$$

Let $S_{k+1 / 2}^{\prime}(4 M, \chi)$ denote the orthogonal complement in $S_{k+1 / 2}(4 M, \chi)$ of the space of theta series in one variable. Note that $S_{k+1 / 2}^{\prime}(4 M, \chi)$ is the entire space $S_{k+1 / 2}(4 M, \chi)$ 
for $k \geq 2$, while for $k=1$ it equals the subspace $V(4 M ; \chi)$ defined in [U93]. In the following we prove:

Theorem 6. Let B, C, $\theta$ be the constants as in Theorem 1. If

$$
f(z)=\sum_{n=1}^{\infty} \rho_{f}(n)(4 \pi n)^{k / 2+1 / 4} e(n z)
$$

is an $L^{2}$-normalized cusp form in $S_{k+1 / 2}^{\prime}(4 M, \chi)$ (cf. (2.1), (2.8)), then for $\varepsilon>0$ and for any $n \geq 1$ whose square part is coprime with $2 M$ we have

$$
\sqrt{n} \rho_{f}(n) \ll_{\epsilon}(k M n)^{\epsilon}\left(\Gamma\left(k+\frac{1}{2}\right)\right)^{-1 / 2} k^{(B+1) / 2} M^{C+1} n^{1 / 4-(1 / 16)(1-2 \theta)} .
$$

Remark 7.1. A similar bound holds for Maass forms. For example, when

$$
f(x+i y)=\sum_{\substack{n \in \mathbb{Z} \\ n \neq 0}} \rho_{f}(n) W_{(n /|n|)(1 / 4), i t}(4 \pi|n| y) e(n x)
$$

is an $L^{2}$-normalized Maass cusp form of weight $\frac{1}{2}$, level $4 M$, and Laplacian eigenvalue $\frac{1}{4}+t^{2}(t \in \mathbb{R})$, one has, for any $n \geq 1$ whose square part is coprime with $2 M$,

$$
\sqrt{n} \rho_{f}(n) \ll_{\epsilon}((1+|t|) M|n|)^{\epsilon}(\operatorname{ch}(\pi t))^{1 / 2}(1+|t|)^{B^{\prime}} M^{C^{\prime}}|n|^{1 / 4-(1 / 16)(1-2 \theta)}
$$

for some positive constants $B^{\prime}$ and $C^{\prime}$. We leave the details to a future work.

Remark 7.2. The first breakthrough in obtaining nontrivial bounds for the Fourier coefficients of half-integral weight cusp forms was achieved by Iwaniec [I87] and reads with slight refinements as (cf. Lemma 2 in [DSp90])

$$
\sqrt{n} \rho_{f}(n) \ll_{\epsilon}(k M n)^{\epsilon}\left(\Gamma\left(k+\frac{1}{2}\right)\right)^{-1 / 2} k^{9 / 2} n^{1 / 4-1 / 28}
$$

with the above normalization and under the above assumptions, providing that $n$ is square-free. Estimates that are valid for all $n$ have been obtained in [B104a], Theorem 1 and Lemma 4.4, which are particularly useful for applications with ternary quadratic forms. Combining (7.2) with Lemma 4.2 in [B104a], we obtain (cf. [B104a, p. 5] for the notation)

$$
r(\operatorname{spn} f, n)-r(f, n) \ll_{\varepsilon} N^{41 / 16} n^{1 / 2-(1 / 16)(1-2 \theta)+\epsilon}
$$

for integers $n$ whose square part is coprime with $N$. 
7.1. Cusp forms and cuspidal automorphic representations. Let $\mathbb{A}_{\mathbb{Q}}$ be the ring of adeles of the rational field $\mathbb{Q}$. Define the additive character $\psi$ of $\mathbb{A}_{\mathbb{Q}}$ such that $\psi_{\infty}(x)=e^{2 \pi i x}$ over $\mathbb{Q}_{\infty}=\mathbb{R}$ and for $x \in \mathbb{Q}_{p}, \psi_{p}(x)=e^{-2 \pi i \hat{x}}$ where $p^{l} \hat{x} \in \mathbb{Z}$ for some integer $l$ and $x-\hat{x} \in \mathbb{Z}_{p}$, the ring of integers of $\mathbb{Q}_{p}$. The additive measures on $\mathbb{Q}_{v}$ are defined to be self-dual with respect to $\psi_{v}$ for $v=p$ or $\infty$.

Let $f(z) \in S_{k+1 / 2}^{\prime}(4 M, \chi)$, then associated to $f(z)$ is a vector $\tilde{\varphi}=t(f)$ in the space of cuspidal automorphic representations of $\widetilde{S L}_{2}\left(\mathbb{A}_{\mathbb{Q}}\right)$, the two-fold cover of $\mathrm{SL}_{2}\left(\mathbb{A}_{\mathbb{Q}}\right)$. Here $\tilde{\varphi}=t(f)$ is a function on $\widetilde{\mathrm{SL}}_{2}\left(\mathbb{A}_{\mathbb{Q}}\right)$ which is continuous and left-invariant under $\mathrm{SL}_{2}(\mathbb{Q})$ and satisfies

$$
\left(\left(\begin{array}{cc}
\sqrt{y} & x / \sqrt{y} \\
0 & 1 / \sqrt{y}
\end{array}\right)\left(\begin{array}{cc}
\cos \theta & \sin \theta \\
-\sin \theta & \cos \theta
\end{array}\right), 1,1, \ldots\right)=y^{k / 2+1 / 4} e^{i(k+1 / 2) \theta} f(x+y i),
$$

where $y>0, x \in \mathbb{R}$, and $-\pi<\theta \leq \pi$. By the strong approximation theorem for $\mathrm{SL}_{2}$, we see that $\tilde{\varphi}=t(f)$ is unique.

It is clear that $\tilde{\varphi}$ decomposes into a sum of $\tilde{\varphi}_{i}=\bigotimes_{v} \tilde{\varphi}_{i, v}$, where each $\tilde{\varphi}_{i}$ is a vector in some irreducible cuspidal representation $\tilde{\pi}_{i}$ of $\widetilde{S L}_{2}\left(\mathbb{A}_{\mathbb{Q}}\right)$. We will first establish the bound (7.2) in the case when $\tilde{\varphi}=t(f)$ itself is a vector $\tilde{\varphi}=\bigotimes_{v} \tilde{\varphi}_{v}$ in an irreducible cuspidal representation $\tilde{\pi}$ of $\widetilde{S L}_{2}\left(\mathbb{A}_{\mathbb{Q}}\right)$.

In [Wa91] a map $S_{\psi}$ is defined from the set of irreducible cuspidal representations of $\widetilde{S L}_{2}\left(\mathbb{A}_{\mathbb{Q}}\right)$ to the set of irreducible automorphic representations of $\operatorname{PGL}_{2}\left(\mathbb{A}_{\mathbb{Q}}\right)$. With our assumption of orthogonality to one-variable theta series, we see that $\pi:=S_{\psi}(\tilde{\pi})$ is a cuspidal representation. Let $\varphi=\bigotimes_{v} \varphi_{v}$ be the unique (up to scalar multiple) new vector in the space of $\pi$ (cf. [Ca73, Sc02]). Define

$$
g(z):=\varphi(\sigma)(c z+d)^{-2 k}, \quad \sigma=\left(\begin{array}{cc}
a & b \\
c & d
\end{array}\right) \in \mathrm{SL}_{2}(\mathbb{R}), \quad z:=\frac{a i+b}{c i+d}
$$

where $\mathrm{SL}_{2}(\mathbb{R})$ is identified with its image in $\operatorname{PGL}_{2}\left(\mathbb{A}_{\mathbb{Q}}\right)$ under the embedding $\sigma \mapsto(\sigma, 1,1, \ldots)$.

Then $g(z)$ is a newform of weight $2 k$, some level $N$, and trivial nebentypus. We can be more precise on the size of $N$.

Lemma 7.1. We have $N \mid(2 M)^{\infty}$ and $N \leq(4 M)^{2}$.

Proof. If $p$ does not divide $2 M$, then $\tilde{\pi}_{p}$ is unramified, thus so is $\pi_{p}$, which implies that $N$ is not divisible by $p$. Let $c(\pi)$ denote the conductor of $\pi$ (cf. [Sc02]), and let $v_{p}$ denote the $p$-adic valuation on $\mathbb{Q}$. Clearly, the conductor of the character $\chi$ over $\mathbb{Q}_{p}^{*}$ is at most $v_{p}(4 M)$. We see from Section 1.3.2 of [Wa81] that the newform corresponding to the representation $\pi \otimes \chi$ has level at most $2 M$, that is, $c\left(\pi_{p} \otimes \chi\right) \leq$ $v_{p}(2 M)$. Then, from the table of conductors of local representations (see [Sc02]), $c\left(\pi_{p}\right) \leq 2 v_{p}(4 M)$, which is equivalent to $N \leq(4 M)^{2}$. 
We adopt the notation (2.1) to the present situation, that is,

$$
g(z)=\sum_{n \geq 1} \rho_{g}(n)(4 \pi n)^{k} e(n z) .
$$

7.2. A theorem from [BM05]. We recall Theorem 4.3 in [BM05]. The statement we need is:

Theorem 7. Let $\pi=S_{\psi}(\tilde{\pi})$. Let $S=\{\infty\} \cup\{p: p \mid 2 M\}$ be a finite set of places. For all square-free integers $D \geq 1$, either $\tilde{W}^{D}(\tilde{\varphi})=0$ or

$$
\frac{\left|\tilde{W}^{D}(\tilde{\varphi})\right|^{2}}{\|\tilde{\varphi}\|^{2}}=\frac{|W(\varphi)|^{2} L\left(\pi \otimes \chi_{D}, \frac{1}{2}\right)}{\|\varphi\|^{2}} \prod_{v \in S} E_{v}\left(\varphi_{v}, \tilde{\varphi}_{v}, \psi_{v}, D\right),
$$

where

$$
E_{v}\left(\varphi_{v}, \tilde{\varphi}_{v}, \psi_{v}, D\right):=\frac{e\left(\varphi_{v}, \psi_{v}\right)}{e\left(\tilde{\varphi}_{v}, \psi_{v}^{D}\right) L_{v}\left(\pi_{v} \otimes \chi_{D}, \frac{1}{2}\right)|D|_{v}}
$$

The notations are as in [BM05] which we will explain along the way. $\chi_{D}$ is the quadratic character $\mathbb{A}_{\mathbb{Q}}^{*} / \mathbb{Q}^{*}$ associated with the quadratic extension $\mathbb{Q}(\sqrt{D})$. $\tilde{W}^{D}$ and $W$ are Whittaker functionals and are related to the Fourier coefficients:

$$
\begin{aligned}
& \tilde{W}^{D}(\tilde{\varphi}):=\int \tilde{\varphi}\left(\left(\begin{array}{cc}
1 & x \\
& 1
\end{array}\right)\right) \psi(-D x) d x=e^{-2 \pi D}(4 \pi D)^{k / 2+1 / 4} \rho_{f}(D), \\
& W(\varphi):=\int \varphi\left(\left(\begin{array}{cc}
1 & x \\
& 1
\end{array}\right)\right) \psi(-x) d x=e^{-2 \pi}(4 \pi)^{k} \rho_{g}(1) .
\end{aligned}
$$

The Petersson norms are related by (cf. (2.8))

$$
\frac{\|\varphi\|^{2}}{\|\tilde{\varphi}\|^{2}}=\frac{\langle g, g\rangle / \operatorname{vol}\left(\Gamma_{0}(N) \backslash \mathbb{H}\right)}{\langle f, f\rangle / \operatorname{vol}\left(\Gamma_{0}(4 M) \backslash \mathbb{H}\right)}=\langle g, g\rangle \frac{\operatorname{vol}\left(\Gamma_{0}(4 M) \backslash \mathbb{H}\right)}{\operatorname{vol}\left(\Gamma_{0}(N) \backslash \mathbb{H}\right)} .
$$

When $v=\infty, \tilde{\varphi}_{v}$ and $\varphi_{v}$ are the lowest weight vectors in $\tilde{\pi}_{\infty}$ and $\pi_{\infty}$, respectively. The constant $E_{v}\left(\varphi_{v}, \tilde{\varphi}_{v}, \psi_{v}, D\right)$ is computed in [BM05]; it equals

$$
E_{\infty}\left(\varphi_{\infty}, \tilde{\varphi}_{\infty}, \psi_{\infty}, D\right)=\frac{1}{2} e^{4 \pi(1-D)} D^{k-1 / 2} \pi^{-k} \Gamma(k) L\left(\pi_{\infty} \otimes \chi_{D}, \frac{1}{2}\right)^{-1} .
$$

Note that

$$
L\left(\pi \otimes \chi_{D}, \frac{1}{2}\right)=L_{\infty}\left(\pi_{\infty} \otimes \chi_{D}, \frac{1}{2}\right) L\left(g \otimes \chi_{D}, \frac{1}{2}\right) l_{2}(g, D),
$$


where

$$
l_{2}(g, D):=\frac{L_{2}\left(g \otimes \chi_{D}, \frac{1}{2}\right)}{L_{2}\left(\pi_{2} \otimes \chi_{D}, \frac{1}{2}\right)}
$$

is the quotient of the 2-factors of $L\left(g \otimes \chi_{D}, s\right)$ and $L\left(\pi \otimes \chi_{D}, s\right)$. Thus from Theorem 7 we have

$$
D\left|\rho_{f}(D)\right|^{2} \ll \frac{\left|\rho_{g}(1)\right|^{2} 4^{k} \Gamma(k)}{\langle g, g\rangle / \operatorname{vol}\left(\Gamma_{0}(N) \backslash \mathbb{H}\right)} M^{-1} L\left(g \otimes \chi_{D}, \frac{1}{2}\right) l_{2}(g, D) \prod_{p \mid 2 M} E_{p}\left(\varphi_{p}, \tilde{\varphi}_{p}, \psi_{p}, D\right) .
$$

The method of [HL94] implies that (cf. [HM04a, Section 2.6], [DFI94b, p. 219])

$$
\frac{\left|\rho_{g}(1)\right|^{2} \Gamma(2 k)}{\langle g, g\rangle / \operatorname{vol}\left(\Gamma_{0}(N) \backslash \mathbb{H}\right)} \ll_{\epsilon}(k N)^{\epsilon} .
$$

We note that by the duplication formula for the Gamma function we have

$$
\frac{4^{k} \Gamma(k)}{\Gamma(2 k)}=\frac{2 \sqrt{\pi}}{\Gamma\left(k+\frac{1}{2}\right)},
$$

so that the above bound is equivalent to

$$
\frac{\left|\rho_{g}(1)\right|^{2} 4^{k} \Gamma(k)}{\langle g, g\rangle / \operatorname{vol}\left(\Gamma_{0}(N) \backslash \mathbb{H}\right)} \ll_{\epsilon}(k N)^{\epsilon}\left(\Gamma\left(k+\frac{1}{2}\right)\right)^{-1}
$$

As $L_{2}\left(g \otimes \chi_{D}, \frac{1}{2}\right) \leq \frac{2}{(\sqrt{2}-1)^{2}}$ and $L_{2}\left(\pi_{2} \otimes \chi_{D}, \frac{1}{2}\right) \geq \frac{2}{(\sqrt{2}+1)^{2}}$, we see that

$$
l_{2}(g, D) \ll 1 .
$$

Therefore in order to obtain an upper bound for $\left|\rho_{f}(D)\right|$, we only need to find upper bounds for $E_{p}\left(\varphi_{p}, \tilde{\varphi}_{p}, \psi_{p}, D\right)$.

7.3. Estimating $\boldsymbol{E}_{\boldsymbol{p}}\left(\boldsymbol{\varphi}_{\boldsymbol{p}}, \tilde{\varphi}_{\boldsymbol{p}}, \psi_{\boldsymbol{p}}, \boldsymbol{D}\right)$. In the definition (7.6) of $E_{v}\left(\varphi_{v}, \tilde{\varphi}_{v}, \psi_{v}, D\right)$, we have

$$
\begin{aligned}
e\left(\varphi_{v}, \psi_{v}\right) & :=\frac{\left\|\varphi_{v}\right\|^{2}}{\left|L_{v}\left(\varphi_{v}\right)\right|^{2}}, \\
e\left(\tilde{\varphi}_{v}, \psi_{v}^{D}\right) & :=\frac{\left\|\tilde{\varphi}_{v}\right\|^{2}}{\left|\tilde{L}_{v}^{D}\left(\tilde{\varphi}_{v}\right)\right|^{2}} .
\end{aligned}
$$


Here $L_{v}$ and $\tilde{L}_{v}^{D}$ are the local $\left(D\right.$-th) Whittaker functionals, $\left\|\varphi_{v}\right\|^{2}$ is the local Hermitian form defined as

$$
\left\|\varphi_{v}\right\|^{2}:=\int\left|L_{v}\left(\pi_{v}\left(\left(\begin{array}{ll}
a & \\
& 1
\end{array}\right)\right) \varphi_{v}\right)\right|^{2} \frac{d a}{|a|_{v}}
$$

and $\left\|\tilde{\varphi}_{v}\right\|^{2}$ is the local Hermitian form defined as

$$
\left\|\tilde{\varphi}_{v}\right\|^{2}:=\frac{|2|_{v}}{2} \sum_{\delta} \int\left|\tilde{L}_{v}^{D \delta}\left(\tilde{\pi}_{v}\left(\left(\begin{array}{ll}
a & \\
& a^{-1}
\end{array}\right)\right) \tilde{\varphi}_{v}\right)\right|^{2} \frac{d a}{|a|_{v}} .
$$

The $\delta$ is summed over the representatives of square classes of $\mathbb{Q}_{v}^{*}$, and $\tilde{L}_{v}^{D \delta}$ are local Whittaker functionals fixed to be compatible with $\tilde{L}^{D}$.

When $v=p$ with $p \mid 2 M, \tilde{\varphi}_{p}$ is a vector with

$$
\tilde{\pi}_{p}(k) \tilde{\varphi}_{p}=\chi_{p}(k) \tilde{\varphi}_{p}, \quad k \in K_{p}(M)
$$

where $K_{p}(M)$ is the subgroup of $\operatorname{SL}_{2}\left(\mathbb{Q}_{p}\right)$ consisting of matrices $\left(\begin{array}{ll}a & b \\ c & d\end{array}\right)$ with $a, d \in \mathbb{Z}_{p}^{*}, b \in \mathbb{Z}_{p}$ and $c \in 4 M \mathbb{Z}_{p}^{*} ; \chi_{p}(k)$ is some unitary character of the group $K_{p}(M)$. We note that the double cover $\widetilde{S L}_{2}$ splits over $K_{v}(M)$, thus we can consider $K_{v}(M)$ as a subgroup of $\widetilde{\mathrm{SL}}_{2}\left(\mathbb{Q}_{v}\right)$.

Lemma 7.2. When $\tilde{\varphi}_{p}$ satisfies equation (7.14), $e\left(\tilde{\varphi}_{p}, \psi_{p}^{D}\right)^{-1} \leq \frac{2}{|2|_{p}}\left(1-p^{-1}\right)^{-1}$ for any $D \in \mathbb{Q}_{p}^{*}$.

Proof. Clearly,

$$
\left\|\tilde{\varphi}_{p}\right\|^{2} \geq \frac{|2|_{p}}{2} \int_{|a|_{p}=1}\left|\tilde{L}_{p}^{D}\left(\tilde{\pi}_{p}\left(\left(\begin{array}{ll}
a & \\
& a^{-1}
\end{array}\right)\right) \tilde{\varphi}_{p}\right)\right|^{2} d a .
$$

When $|a|_{p}=1,\left(\begin{array}{cc}a & \\ & a^{-1}\end{array}\right) \in K_{p}(M)$ and we get, for some unitary character $\chi^{\prime}$,

$$
\tilde{\pi}_{p}\left(\left(\begin{array}{cc}
a & \\
& a^{-1}
\end{array}\right)\right) \tilde{\varphi}_{p}=\chi^{\prime}(a) \tilde{\varphi}_{p} .
$$

As the Whittaker functional $\tilde{L}^{D}$ is a linear form, we see that the integrand is identically $\left|\tilde{L}_{p}^{D}\left(\tilde{\varphi}_{p}\right)\right|^{2}$. The integral thus gives $\left(1-p^{-1}\right)\left|\tilde{L}_{p}^{D}\left(\tilde{\varphi}_{p}\right)\right|^{2}$. From the definition of $e\left(\tilde{\varphi}_{p}, \psi_{p}^{D}\right)$ we get the lemma.

The vector $\varphi_{p}$ is a new vector in $\pi_{p}$. Let $F_{p}(x)$ be its image in the Kirillov model of $\pi_{p}$. Then $L_{p}\left(\varphi_{p}\right)=F_{p}(1)$ which we will assume to be 1 , and 


$$
\left\|\varphi_{p}\right\|^{2}=\int\left|F_{p}(x)\right|^{2} \frac{d x}{|x|}
$$

Looking through the table of $F_{p}(x)$ in [Sc02], we see either $\pi_{p}$ is an unramified representation or $\left|F_{p}(x)\right| \leq|x|^{1 / 2} \operatorname{char}_{\mathbb{Z}_{p}}(x)$. In the latter case we get $\left\|\varphi_{p}\right\|^{2} \leq 1$. In the former case we computed $\left\|\varphi_{p}\right\|^{2}$ in [BM05] and obtained

$$
\left\|\varphi_{p}\right\|^{2}=\left(1+p^{-1}\right)\left|1-p^{-2 s-1}\right|^{-2} .
$$

Here $s$ is a purely imaginary parameter (by the Ramanujan conjecture established by Deligne) associated to $\pi_{p}$. Thus we have

Lemma 7.3. $e\left(\varphi_{p}, \psi_{p}\right) \leq\left(1+p^{-1}\right)\left(1-p^{-1}\right)^{-2}$.

Clearly, $|D|_{p} \geq p^{-1}$. Looking through the table of $L\left(\pi_{p}, \frac{1}{2}\right)$ in [Go70] we get

$$
L\left(\pi_{p} \otimes \chi_{D}, \frac{1}{2}\right) \geq\left(1+p^{1 / 2}\right)^{-2} .
$$

Combining this with (7.6) and Lemmata 7.2-7.3 we get

Lemma 7.4. $E_{p}\left(\varphi_{p}, \tilde{\varphi}_{p}, \psi_{p}, D\right) \leq \frac{2}{|2|_{p}}\left(1+p^{-1}\right)\left(p+p^{1 / 2}\right)^{2}\left(1-p^{-1}\right)^{-3}$.

7.4. Concluding Theorem 6. The above Lemma gives

$$
\prod_{p \mid 2 M} E_{p}\left(\varphi_{p}, \tilde{\varphi}_{p}, \psi_{p}, D\right) \ll_{\varepsilon} M^{2+\varepsilon}
$$

Combining this estimate with (7.11), (7.12), (7.13), and Lemma 7.1 we obtain

$$
D\left|\rho_{f}(D)\right|^{2} \ll_{\epsilon}(k M)^{\epsilon}\left(\Gamma\left(k+\frac{1}{2}\right)\right)^{-1} M L\left(g \otimes \chi_{D}, \frac{1}{2}\right) .
$$

By Theorem 1 and Lemma 7.1,

$$
L\left(g \otimes \chi_{D}, \frac{1}{2}\right) \ll_{\epsilon}(k M D)^{\epsilon} k^{B} M^{2 C} D^{1 / 2-(1 / 8)(1-2 \theta)} .
$$

Thus we get, for square-free $D$,

$$
\sqrt{D} \rho_{f}(D) \ll_{\epsilon}(k M D)^{\epsilon}\left(\Gamma\left(k+\frac{1}{2}\right)\right)^{-1 / 2} k^{B / 2} M^{C+1 / 2} D^{1 / 4-(1 / 16)(1-2 \theta)} .
$$

From the theory of the Shimura correspondence [Sh73, Theorem 1.9], we see that for $n=D t^{2}$ with $D$ square-free and $(t, 2 M)=1$, we have 


$$
\sqrt{n} \rho_{f}(n)=\sqrt{D} \rho_{f}(D) \sum_{r s=t} \frac{\mu(r) \chi(r)}{\sqrt{r}}\left(\frac{-1}{r}\right)^{k}\left(\frac{D}{r}\right) \lambda(s),
$$

where $\lambda(s)$ is the $s$-th Hecke eigenvalue of a fixed newform of weight $2 k$. By Deligne's bound, $|\lambda(s)| \leq \tau(s) \leq \tau(n)$ which shows that (7.15) remains valid if $D$ is replaced by any positive integer $n$ whose square part is coprime with $2 M$.

Now an arbitrary $f(z)$ with $\langle f, f\rangle=1$ is a linear combination $\sum_{i} b_{i} f_{i}(z)$, where

$$
f_{i}(z)=\sum \rho_{f_{i}}(n)(4 \pi n)^{k / 2+1 / 4} e(n z),
$$

$\left\langle f_{i}, f_{j}\right\rangle=\delta_{i, j}$ (thus $\sum_{i}\left|b_{i}\right|^{2}=1$ ), and the Fourier coefficients $\rho_{f_{i}}(n)$ satisfy the bound of (7.15). By Cauchy-Schwarz,

$$
\left|\rho_{f}(n)\right|^{2} \leq \sum_{i}\left|\rho_{f_{i}}(n)\right|^{2}
$$

and by Theorem 4.2.1 of [ $\mathrm{Ra} 77]$, the dimension of $S_{k+1 / 2}^{\prime}(4 M, \chi)$ is at most

$$
\frac{k+\frac{1}{2}}{12}\left[\mathrm{SL}_{2}(\mathbb{Z}): \Gamma_{0}(4 M)\right] \ll_{\varepsilon} k M^{1+\varepsilon},
$$

therefore we conclude the bound (7.2).

\section{References to Appendix 2}

[BM05] Baruch E. M., Mao Z.: Central values of automorphic $L$-functions. To appear in Geom. Funct. Anal.

[B104a] Blomer V.: Uniform bounds for Fourier coefficients of theta-series with arithmetic applications. Acta Arith. 114 (2004), 1-21

[Ca73] Casselman W.: On some results of Atkin and Lehner. Math. Ann. 201 (1973), 301-314

[DFI94b] Duke W., Friedlander J., Iwaniec H.: Bounds for automorphic $L$-functions. II. Invent. Math. 115 (1994), 219-239

[DSp90] Duke W., Schulze-Pillot R.: Representation of integers by positive ternary quadratic forms and equidistribution of lattice points on ellipsoids. Invent. Math. 99 (1990), $49-57$

[Go70] Godement R.: Notes on Jacquet-Langlands theory. The Institute for Advanced Study (Princeton), 1970

[HM04a] Harcos G., Michel P.: The subconvexity problem for Rankin-Selberg $L$-functions and equidistribution of Heegner points. II. Invent. Math. 163 (2006), 581-655

[HL94] Hoffstein J., Lockhart P.: Coefficients of Maass forms and the Siegel zero. Ann. of Math. 140 (1994), 161-181

[I87] Iwaniec H.: Fourier coefficients of modular forms of half-integral weight. Invent. Math. 87 (1987), 385-401

[Ra77] Rankin R. A.: Modular forms and functions. Cambridge University Press, Cambridge-New York-Melbourne 1977

[Sc02] Schmidt R.: Some remarks on local newforms for GL(2). J. Ramanujan Math. Soc. 17 (2002), 115-147

[Sh73] Shimura G.: On modular forms of half integral weight. Ann. of Math. 97 (1973), $440-481$ 
[U93] Ueda M.: On twisting operators and newforms of half-integral weight. Nagoya Math. J. 131 (1993), 135-205

[Wa81] Waldspurger J.-L.: Sur les coefficients de Fourier des formes modulaires de poids demi-entier. J. Math. Pures Appl. 60 (1981), 375-484

[Wa91] Waldspurger J.-L.: Correspondances de Shimura et quaternions. Forum Math. 3 (1991), 219-307

\section{References}

[B104b] Blomer V.: Shifted convolution sums and subconvexity bound for automorphic $L$-functions. Int. Math. Res. Not. 2004, 3905-3926

[B63] Burgess D. A.: On character sums and $L$-series. Proc. London Math. Soc. 12 (1962), 193-206; II, ibid. 13 (1963), 524-536

[By96] Bykovskii V. A.: A trace formula for the scalar product of Hecke series and its applications. Translated in J. Math. Sci (New York) 89 (1998), 915-932

[Co03] Cogdell J.: On sums of three squares. Les XXIIèmes Journées Arithmetiques (Lille, 2001). J. Théor. Nombres Bordeaux 15 (2003), 33-44

[CoPSS] Cogdell J., Piatetskii-Shapiro I. I., Sarnak P.: Estimates for Hilbert modular $L$-functions and applications; in preparation

[CI00] Conrey B., Iwaniec H.: The cubic moment of central values of automorphic $L$-functions. Ann. of Math. 151 (2000), 1175-1216

[DI82] Deshouillers J.-M., Iwaniec H.: Kloosterman sums and Fourier coefficients of cusp forms. Invent. Math. 70 (1982/83), 219-288

[DFI93] Duke W., Friedlander J., Iwaniec H.: Bounds for automorphic $L$-functions. Invent. Math. 112 (1993), 1-8

[DFI94a] Duke W., Friedlander J., Iwaniec H.: A quadratic divisor problem. Invent. Math. 115 (1994), 209-217

[DFI02] Duke W., Friedlander J., Iwaniec H.: The subconvexity problem for Artin $L$-functions. Invent. Math. 149 (2002), 489-577

[H02] Harcos G.: Uniform approximate functional equation for principal $L$-functions. Int. Math. Res. Not. 2002, no. 18, 923-932; Erratum, ibid. 2004, no. 13, 659-660

[H03a] Harcos G.: An additive problem in the Fourier coefficients of Maass forms. Math. Ann. 326 (2003), 347-365

[H03b] Harcos G.: New bounds for automorphic $L$-functions. Ph.D. thesis, Princeton University, 2003

[HM04a] Harcos G., Michel P.: The subconvexity problem for Rankin-Selberg $L$-functions and equidistribution of Heegner points. II. Invent. Math. 163 (2006), 581-655

[HM04b] Harcos G., Michel P.: An improved subconvexity bound for twisted $L$-functions. Unpublished

[HB78] Heath-Brown D. R.: Hybrid bounds for Dirichlet $L$-functions. Invent. Math. 47 (1978), 149-170

[I87] Iwaniec H.: Fourier coefficients of modular forms of half-integral weight. Invent. Math. 87 (1987), 385-401

[IS00] Iwaniec H., Sarnak P.: Perspectives in the analytic theory of $L$-functions. Geom. Funct. Anal. 2000, Special Volume, Part II, 705-741

[J92] Jutila M.: Transformations of exponential sums. Proceedings of the Amalfi Conference on Analytic Number Theory (Maiori 1989). Univ. Salerno, Salerno 1992, $263-270$ 
[J96] Jutila M.: A variant of the circle method. Sieve methods, exponential sums and their applications in number theory, 245-254. Cambridge University Press, 1996

[J99] Jutila M.: Convolutions of Fourier coefficients of cusp forms. Publ. Inst. Math. (Beograd) (N.S.) 65(79) (1999), 31-51

[K03] Kim H.: Functoriality for the exterior square of $\mathrm{GL}_{4}$ and the symmetric fourth of $\mathrm{GL}_{2}$ (with Appendix 1 by D. Ramakrishnan and Appendix 2 by H. Kim and P. Sarnak). J. Amer. Math. Soc. 16 (2003), 139-183

[KS03] Kim H., Sarnak P.: Appendix: Refined estimates towards the Ramanujan and Selberg Conjectures. J. Amer. Math. Soc. 16 (2003), 175-181

[KS02] Kim H., Shahidi F.: Cuspidality of symmetric powers with applications. Duke Math. J. 112 (2002), 177-197

[KMV02] Kowalski E., Michel P., VanderKam J.: Rankin-Selberg $L$-functions in the level aspect. Duke Math. J. 114 (2002), 123-191

[M04a] Michel P.: The subconvexity problem for Rankin-Selberg $L$-functions and equidistribution of Heegner points. Ann. of Math. 160 (2004), 185-236

[M04b] Michel P.: Analytic number theory and families of $L$-functions. Park City Lecture Notes (2004, to appear), available at http://www.math.univ-montp2.fr/ michel/

[Ra90] Rankin R. A.: Diagonalizing Eisenstein series. I. In: Analytic number theory (Allerton Park, IL, 1989), 429-450. Progr. Math. 85 (1990). Birkhäuser Boston, Boston, MA

[Ra93] Rankin R. A.: Diagonalizing Eisenstein series. II. A tribute to Emil Grosswald. In: Number Theory and Related Analysis, 525-537. Contemp. Math. 143 (1993). Amer. Math. Soc. Providence, RI

[Ra92] Rankin R. A.: Diagonalizing Eisenstein series. III. In: Discrete Groups and Geometry (Birmingham, 1991), 196-208. London Math. Soc. Lecture Note Ser. 173 (1992). Cambridge Univ. Press, Cambridge

[Ra94] Rankin R. A.: Diagonalizing Eisenstein series. IV. In: The Rademacher Legacy to Mathematics (University Park, PA, 1992), 107-118. Contemp. Math. 166 (1994). Amer. Math. Soc. Providence, RI

[S01] Sarnak P.: Estimates for Rankin-Selberg $L$-functions and quantum unique ergodicity. J. Funct. Anal. 184 (2001), 419-453

Received November 23, 2004

Mathematisches Institut, Bunsenstrasse 3-5, D-37073 Göttingen, Germany

blomer@uni-math.gwdg.de

The University of Texas at Austin, Mathematics Department, 1 University Station C1200, Austin, TX 78712-0257, USA

gharcos@math.utexas.edu

I3M, UMR CNRS 5149, Université Montpellier II CC 051, 34095 Montpellier Cedex 05, France

michel@math.univ-montp2.fr

Department of Mathematics and Computer Science, Rutgers University, Newark, NJ 071021811, USA

zmao@andromeda.rutgers.edu 